\title{
A numerical study of breaking waves
}

\author{
Chiyoon Song and Ana I. Sirviente ${ }^{\text {a) }}$ \\ Department of Naval Architecture and Marine Engineering, The University of Michigan, Ann Arbor, \\ Michigan 48109-2145
}

(Received 7 July 2003; accepted 18 March 2004; published online 4 June 2004)

\begin{abstract}
This numerical study explores the physical processes involved in breaking waves. The two-dimensional, incompressible, unsteady Navier-Stokes equations are solved in sufficiently refined grids to capture viscous and capillary effects. The immiscible interface, characterized by a jump in density and viscosity, is embedded in the domain and a hybrid front tracking/capturing method is used to characterize the moving interface of this multiphase flow. A parametric study is conducted to assess the role of surface tension, Reynolds number, density, and viscosity on the breaking process, as well as their role in the vorticity redistribution and energy dissipation beneath the surface. (C) 2004 American Institute of Physics. [DOI: 10.1063/1.1738417]
\end{abstract}

\section{INTRODUCTION}

Free surface flows have applications in very diverse areas such as oceanography, engineering and fundamental physics, among others. Liquid atomizers, such as those used by internal combustion engines, aerosol generators for drug delivery or ink-jet printers are few examples of engineering applications where interface between immiscible fluids are present and the understanding of their initial breakup is crucial to their optimization. In oceanography, the study of breaking waves is of significant importance to better understand the mixing processes that take place in the upper layer of the oceans and play a significant role in the transfer of mass, heat and momentum. Remote observations of the ocean surface by radar can provide directional data on wave heights and wind speeds. The appropriate interpretation of this data is linked to an in-depth understanding of the wave breaking processes, and it is also crucial for the safety of vessels and structures at sea. In severe sea conditions, tremendous damage can be caused by the large forces induced by the breaking of waves.

Wave breaking in an infinite domain implies that there is no direct or indirect effect on the process of breaking from solid boundaries (i.e., bed topography). Deep water breaking waves are characterized by "white water" falling down the front face of the wave. Plunging waves are dramatic and energetic with a characteristic overturning of the wave and consequent formation of a jet that plunges down into the water. Wave breaking, in general, is an extremely complex unsteady, nonlinear, highly turbulent phenomenon, involving two fluids of very different properties. This physical process has constituted an important challenge to both experimental and numerical techniques. The observation and detailed quantification of a highly unsteady and rapidly evolving phenomena is not a trivial matter either for field or laboratory measurements. While experiments in the laboratory are better controlled and allow for better conditions than in the

\footnotetext{
a) Author to whom correspondence should be addressed; Telephone: (734) 647-9411; fax: (734) 936-8820. Electronic mail: asirv@engin.umich.edu
}

field, the results from small scales with significant surface tension and viscosity effects are not easily scalable to sea or full-scale conditions. This is especially complex when one of the boundary conditions needs to be applied on a spatially moving boundary. On the other hand, numerical studies face the challenge of achieving adequate spatial and temporal resolution to resolve the small scale viscous and capillary effects.

In their review of wave breaking in deep waters, Banner and Peregrine ${ }^{1}$ subdivide laboratory studies into those concerned with detecting breaking, and those investigating the kinematics of the breaking waves. The first group of studies are concerned with the characterization of the instability mechanism leading to breaking as well as the study of simple criteria to identify breaking (Duncan, ${ }^{2}$ Melville, ${ }^{3,4}$ Su et al.,${ }^{5}$ Ramberg et al., ${ }^{6}$ and Ebuchi et al. $^{7}$ among others). The idea of using simple local wave parameters to detect breaking has been proposed by some researchers. The measurements by Melville and Rapp ${ }^{8}$ show changes in wave slope as breaking is approached. Kjeldsen and Myrhaug, ${ }^{9}$ Bonmarin and Ramamonjiarsoa ${ }^{10}$ and Bonmarin ${ }^{11}$ use four parameters to define the asymmetry of the wave profile and study the deformation to breaking of deep water waves. Bonmarin ${ }^{11}$ argues that the shape of steep progressive waves undergoes a continuous change of profile both in space and time, and that such shape change can take place very rapidly. A very detailed study of the kinematics of deep water breaking is presented by Rapp and Melville. ${ }^{12}$ It provides measurements of not only surface motion and momentum flux, but also breaking induced currents, turbulent fluctuations and dissipation of energy. Skyner ${ }^{13}$ and Perlin et al. ${ }^{14}$ have reported full-field velocity measurements of plunging breakers. Skyner's ${ }^{13}$ work compares the numerical predictions from a boundary integral method with the particle image velocimetry (PIV) measurements of the internal kinematics of a deep water plunging wave. His results show good agreement throughout, except on the approach to breaking where the wave crests did not match and showed earlier plunging in the numerical simulation. Perlin et al. ${ }^{14}$ measured the evolution of a plung- 


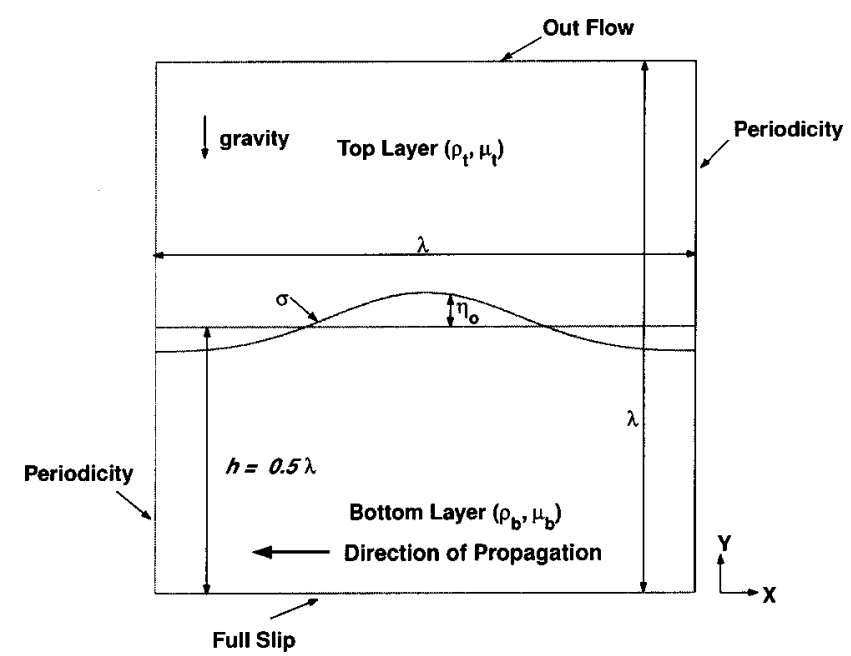

FIG. 1. Schematic of the computational domain showing boundary conditions.

ing breaker using nonintrusive surface elevation measurements, PIV and particle tracking velocimetry (PTV) techniques. The study focused on the surface profile to the point of jet impingement and on the flow field to the point of jet formation.

The onset of breaking of spilling breakers involves small-scale capillary waves. These appear in the leeward side of the toe of a well define bore-like structure, which eventually evolves into irregular distortions of the free surface. These breaking patterns have been observed experimentally in wind-induced short-wavelength spilling breakers by Schooley, ${ }^{15}$ Okuda, ${ }^{16}$ and Ebuchi et al. ${ }^{7}$ and in mechanically generated breakers by Lin and Rockwell ${ }^{17}$ and Duncan ${ }^{18-21}$ among others. The latter studies focus on the evolution of the surface profile and the measurements of the flow field at the crest of the breaker with PIV.

Longuet-Higgins, ${ }^{22}$ in his review of breaking waves, presents a detailed overview concerning crest instabilities of the almost highest Stokes wave. The nonlinear development of the wave may result in overturning of the crest or else it might transition to a lower amplitude periodic wave, and then to a quasiperiodic recurrence, depending on the characteristics of the initial disturbance. Longuet-Higgins and Cocklet $^{23}$ were the first to simulate the evolution of twodimensional overturning waves based on potential flow theory. Fully nonlinear time-stepping numerical techniques have been applied more recently for the numerical prediction of two dimensional plunger breakers (Dold and Peregrine, ${ }^{24}$ Dommermuth et al. ${ }^{25}$ Grilli and Svendsen, ${ }^{26}$ and Skyner et $a .^{27}$ ). These techniques are limited to the early stages of breaking. Comparisons of some of these numerical results with experimental data have been shown in the literature (Dommermuth et al. ${ }^{25}$ Skyner et al. ${ }^{27}$ and Skyner ${ }^{13}$ ). The simulations seem to predict the plunging of the wave closer to the wavemaker in contrast to what is observed in the experiments. Tulin ${ }^{28}$ studied the effects of surface tension on a breaker by performing a potential flow simulation of the evolution of waves toward breaking. Their results showed that, as the surface tension effects become more important, the tip of the jet goes from being rounded to being pressed and is replaced by a bulge at the crest of the wave. Similar results were presented by Ceniceros and Hou, ${ }^{29}$ who also included weak viscous effects by using a modified boundary integral formulation. The early stages of breaking of a short gravity waves, as described by Longuet-Higgins, ${ }^{30}$ are characterized by the presence of small-scale capillary waves (referred to as Type I by Longuet-Higgins ${ }^{31}$ ) that serve as vorticity generators to the roller beneath the wave crest. These Type I capillary waves are followed by longer capillaries (Type II), instability waves (Longuet-Higgins ${ }^{31}$ ), occurring in a shear layer immediately below the free surface and induced by the vorticity shed by the Type I capillary waves. The work of Mui and Dommermuth ${ }^{32}$ and Ceniceros ${ }^{33}$ are among the few attempts to numerically simulate the formation and evolution of capillary waves including both surface tension and viscosity effects.

Numerical simulations of breaking waves post impact of the impinging jet are a challenge to numerical schemes and consequently rare in the literature (i.e., Monaghan et al., ${ }^{34}$ Chen et al. ${ }^{35}$ and Iafrati and Campana ${ }^{36}$ ). Monaghan et al. ${ }^{34}$ used the smoothed particle hydrodynamics method to simulate splash up (i.e., the post-breaking phase), Chen et al. ${ }^{35}$ solved the Navier-Stokes equations and used volume of fluid method to resolve the two-phase flow. Their numerical results show that more than $80 \%$ of the total wave energy present prior to wave breaking is dissipated within three wave periods. They also discuss the vortex-like motion induced beneath the surface due to the breaking process. Iafrati and Campana ${ }^{36}$ used a Level-Set technique to simulate the two-dimensional breaking wave generated by a hydrofoil moving beneath the free surface. They used a heterogeneous unsteady domain decomposition approach by using a potential flow model to simulate the flow about the body and solving the Navier-Stokes equations in the free surface region. In their study, they analyzed the surface tension effects by progressively reducing the length scale while keeping Froude and Reynolds number constant.

The purpose of this study is to contribute to the understanding of the hydrodynamic processes involved in wave breaking. While a considerable amount of work has been done related to the study of breaking waves, few studies entail surface tension and viscosity effects. To that end, twodimensional, incompressible, unsteady Navier-Stokes equations are solved on sufficiently fine grids to capture viscous and capillary effects. The immiscible interface, characterized by a jump in density and viscosity, is embedded in the domain and a finite difference/front tracking method is used to characterize the moving interface of this multiphase flow. In this work, a parametric study is conducted to assess the role of surface tension, Reynolds number, density ratio, viscosity ratio and initial conditions on the breaking processes, as well as their role in the vorticity redistribution beneath the surface and energy dissipation.

In what follows, Sec. II of the present paper describes the computational method used and discusses the interface tracking technique used in these simulations. In Sec. III, the numerical results are presented and discussed. In that section, results are organized as a comparative study based on the 

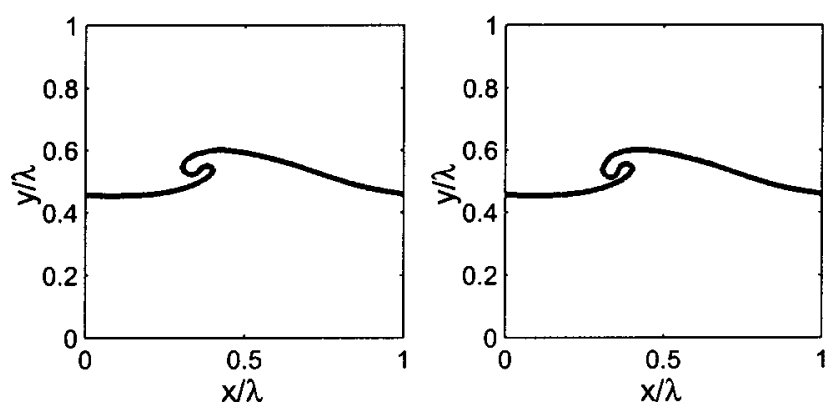

effect of each of the governing nondimensional parameters that define this problem. Finally, conclusions are presented in Sec. IV.

\section{COMPUTATIONAL METHOD AND PROBLEM FORMULATION}

\section{A. Numerical formulation}

The numerical methodology followed in this investigation involves the simulation of incompressible flow of two immiscible fluids. The interface separating both fluids is characterized by the surface tension $(\sigma)$ and the difference of density $(\rho)$ and viscosity $(\mu)$ of the top and bottom fluids. The governing equations used are the Navier-Stokes equations along with the conservation of mass and the incompressibility condition. In this study, the problem is simplified by assuming it two dimensional (2D). Based on a coordinate system where the $x$ axes represents the streamwise, or wave propagation direction and the $y$ axis represents the normal direction, the general conservative form of the 2D NavierStokes equations is as follows:

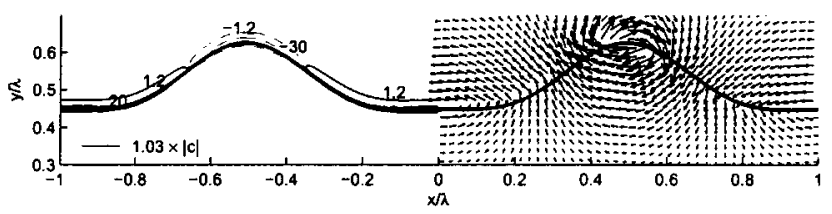

(a) $\tau=0.0164$

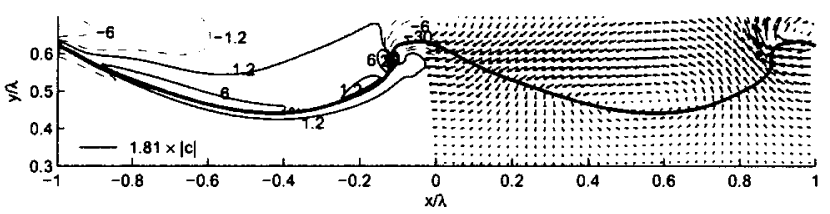

(c) $\tau=0.4578$

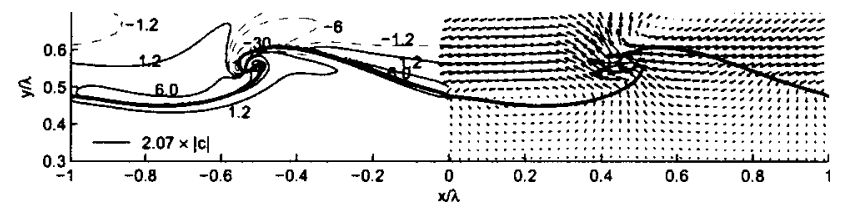

(e) $\tau=0.6776$

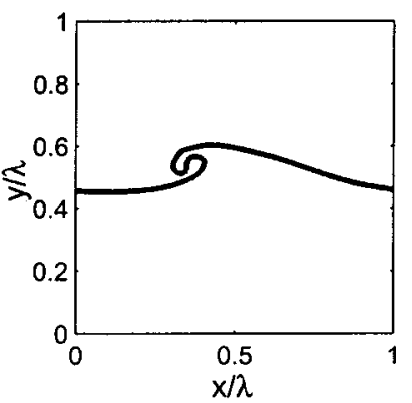

FIG. 2. Comparison of wave profile for different grid resolutions. 480 $\times 480$ (left), $512 \times 512$ (middle), 544 $\times 544 \quad$ (right) $\quad(\operatorname{Re}=350, \quad B o=224$, density ratio $=0.028$, viscosity ratio $=0.3$, and $\mathrm{s}=0.5654$ ).

$$
\begin{aligned}
\frac{\partial \rho u}{\partial t}+ & \frac{\partial}{\partial x}\left(\rho u^{2}\right)+\frac{\partial}{\partial y}(\rho u v) \\
= & -\frac{\partial p}{\partial x}+\frac{\partial}{\partial x}\left(2 \mu \frac{\partial u}{\partial x}\right)+\frac{\partial}{\partial y}\left(\mu\left(\frac{\partial v}{\partial x}+\frac{\partial u}{\partial y}\right)\right) \\
& -\int_{S} \sigma \kappa \mathbf{n} \delta\left(\mathbf{x}-\mathbf{x}_{\mathbf{f}}\right) d S \cdot \mathbf{i}_{\mathbf{x}}+\rho g_{x}, \\
\frac{\partial \rho v}{\partial t}+ & \frac{\partial}{\partial x}(\rho u v)+\frac{\partial}{\partial y}\left(\rho v^{2}\right) \\
= & -\frac{\partial p}{\partial y}+\frac{\partial}{\partial x}\left(\mu\left(\frac{\partial v}{\partial x}+\frac{\partial u}{\partial y}\right)\right)+\frac{\partial}{\partial y}\left(2 \mu \frac{\partial v}{\partial y}\right) \\
& -\int_{S} \sigma \kappa \mathbf{n} \delta\left(\mathbf{x}-\mathbf{x}_{\mathbf{f}}\right) d S \cdot \mathbf{i}_{\mathbf{y}}+\rho g_{y},
\end{aligned}
$$

where the components of the velocity vector, $\mathbf{u}$, in the

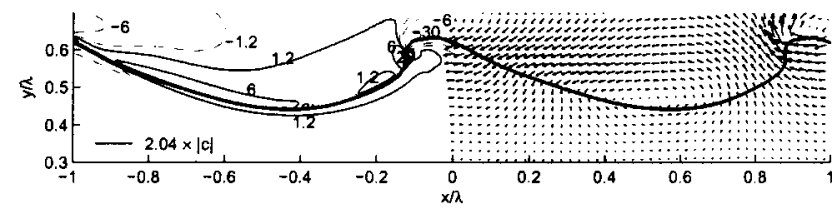

(b) $\tau=0.4189$

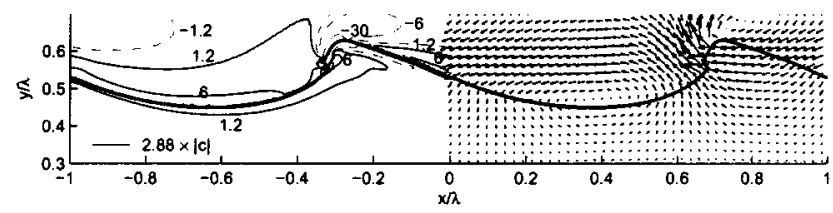

(d) $\tau=0.5813$

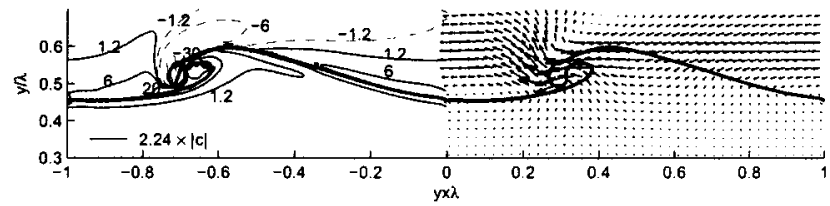

(f) $\tau=0.8490$

FIG. 3. Time sequence of free surface profiles showing the velocity field and the vorticity contours $(\operatorname{Re}=350, B o=224$, density ratio $=0.02$, viscosity ratio $=0.5$, and $\varsigma=0.5581)$. Positive vorticity contour $\left(\omega^{+}\right)---$, negative vorticity contour $\left(\omega^{-}\right)---$. In subplot (a) $\omega^{+}=20,1.2$ and $\omega^{-}=-30,-1.2$, in other subplots $\omega^{+}=20,6,1.2$ and $\omega^{-}=-30,-6,-1.2$. 


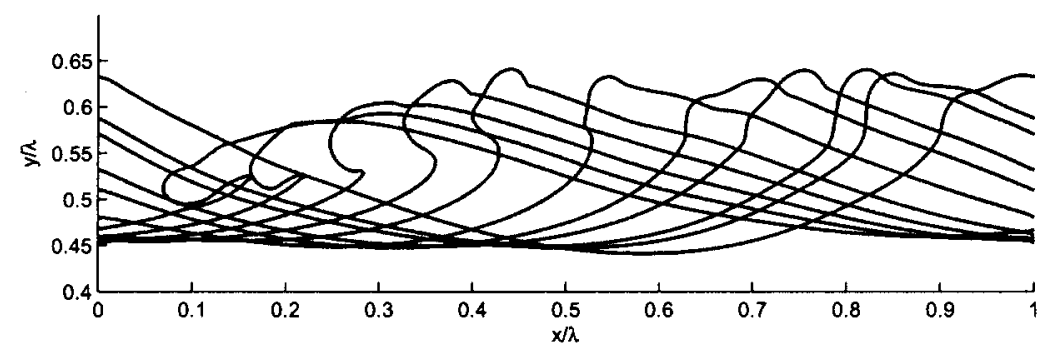

(a) $B o=597, \tau=0.4152,0.4912,0.5204,0.5907,0.6429,0.7193,0.8129,0.8714$, $0.9299,0.9825,1.0357$ (from right).

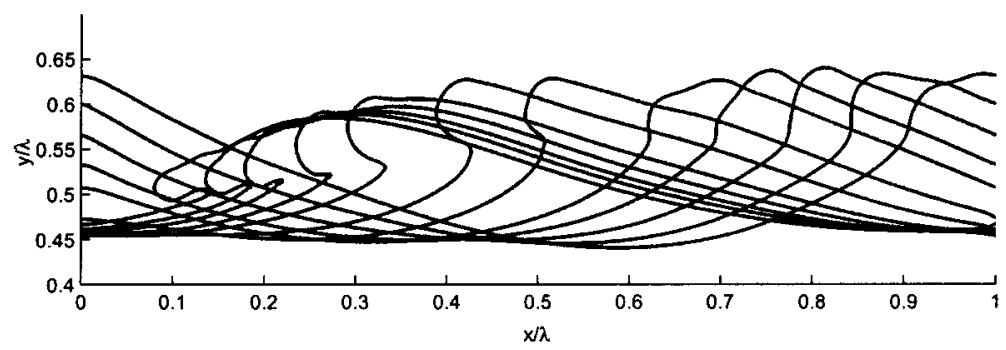

(b) $B o=275, \tau=0.4097,0.4682,0.5350,0.5853,0.6381,0.7376,0.8137,0.8868$, $0.9308,0.9659,0.9984,1.0818$ (from right).

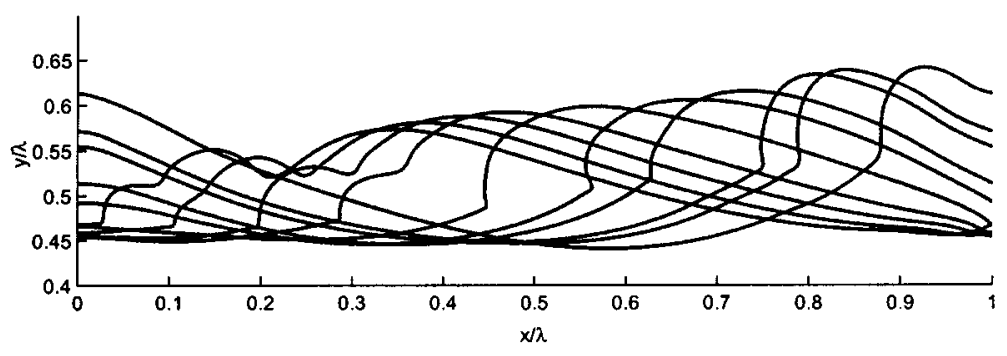

(c) $B o=38, \tau=0.4208,0.4972,0.5268,0.6038,0.6512,0.7147,0.8285,0.8877$,
FIG. 4. Time sequence of free surface profiles for different Bond numbers $(\operatorname{Re}=350$, density ratio $=0.028$, viscosity ratio $=0.5$, and $\varsigma=0.5507)$.

$0.9469,1.002$ (from right).

streamwise and normal directions are $u$ and $v$, respectively, $p$ is the pressure, $\rho$ and $\mu$ are the discontinuous density and viscosity fields, respectively, and $t$ is time. The last term on the right-hand side of Eqs. (1) and (2) represents the contribution to the momentum equations from body forces and the only one considered herein is due to gravity, $g$. The fourth term on the right-hand side represents the contribution from the contact or surface forces acting on the interface, where $\kappa$ is the interface curvature and $\mathbf{n}$, is the unit vector normal to the wave interface with components $\mathbf{i}_{\mathbf{x}}$ in the streamwise direction and $\mathbf{i}_{y}$ in the normal direction. The integral is over the entire interface, $S$, and $\delta$ is a delta function that limits the contribution of the surface tension to the free surface. The interface position is identified by $\mathbf{x}_{\mathbf{f}}$, and $\mathbf{x}$ represents the point at which the equation is evaluated. The proper stress boundary condition at the interface is implicitly contained in these equations.

The conservation of mass for incompressible fluids can be expressed as

$\nabla \cdot \mathbf{u}=0$ since the density of both immiscible fluids remains constant (i.e., the total derivative of density is zero) and the velocity field is divergence free.

The equations of state of the density and viscosity can be expressed as follows:

$$
\frac{D \rho}{D t}=0, \quad \frac{D \mu}{D t}=0
$$

since the viscosity of both immiscible fluids, similar to the density field, also remains constant. The combination of the incompressibility condition [Eq. (3)] and the momentum equations [Eqs. (1) and (2)] leads to the following pressure equation:

$$
\nabla \cdot\left(\frac{1}{\rho}\right) \nabla p=\frac{R}{\rho},
$$

where $R$ represents the divergence of the vector form of the momentum equations when excluding the pressure term.

The system of equations formed by the Navier-Stokes equations, the continuity equation and the pressure equation 


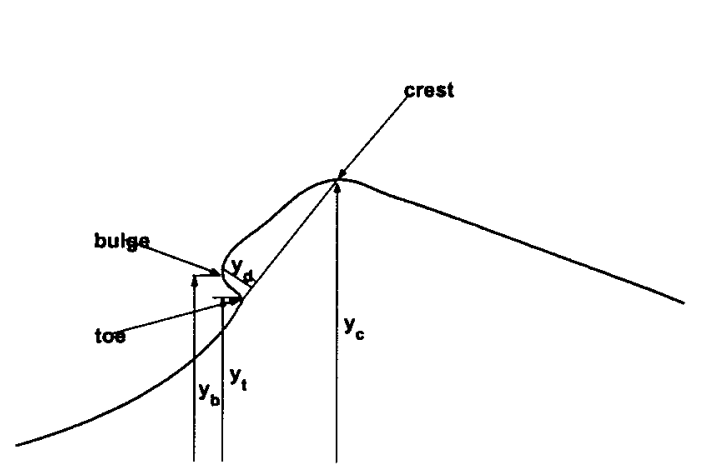

(a) nomenclature

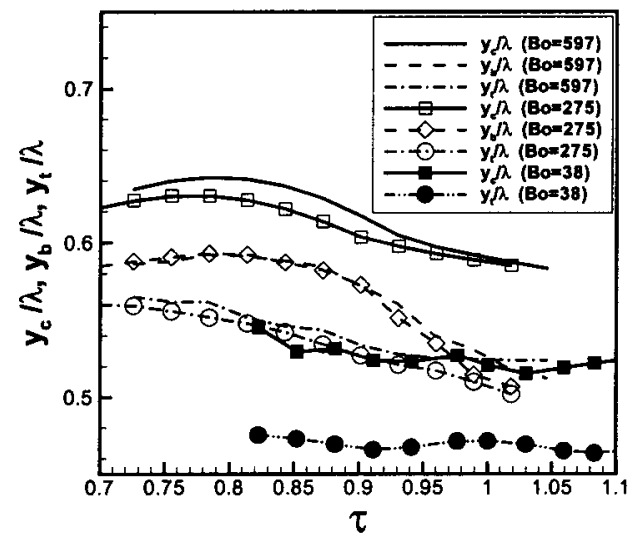

(c) $y_{c}, y_{b}, y_{t}(B o=38,275,597)$

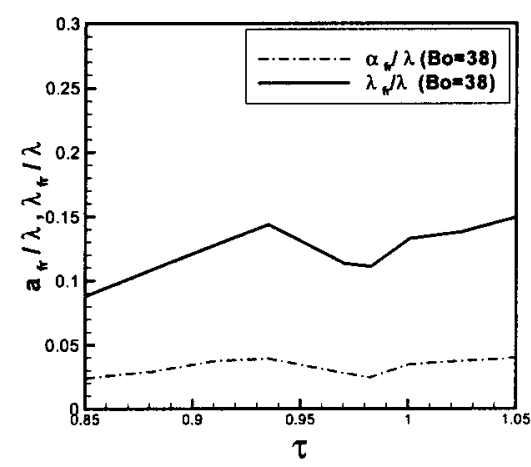

(b) $a_{f r}, \lambda_{f r}(B o=38)$

FIG. 5. Definition and time evolution of geometrical wave profile parameters.

derived above, is discretized in space using a second order central finite difference scheme on a staggered grid (Harlow and Shannon, ${ }^{37}$ Harlow and $\left.W \mathrm{Ch}^{38}\right)$. Time integration is accomplished by using an explicit first order fractional-step or projection type method $\left(\right.$ Chorin $^{39}$ and $\left.\operatorname{Teman}^{40}\right)$. The Navier-Stokes equations, the continuity equation and the pressure equation are solved following a method similar to Tauber et al. ${ }^{41}$ In order to make the numerical scheme computationally efficient, convergence is accelerated by introducing a multigrid approach (Adams ${ }^{42}$ ) to solve the pressure equation.

\section{B. Numerical treatment of the interface}

The main difficulties associated with the numerical study of multiphase flows come from the fact that not only the wavy surface needs to be predicted and simulated but also the viscous and surface tension effects need to be taken into consideration for the technique to be reliable. A comprehensive review of direct numerical simulation of free surface flows can be found in Scardovelli and Zaleski. ${ }^{43}$

In the past, attempts have mostly been made at solving the governing equations in one fluid where boundary-fitted grids are used and readjusted every time the free surface is moved (interface tracking methods). This approach is com- plex and computationally expensive. The simulation of wave breaking presents an obvious major challenge for such techniques. A variation of the so-called interface-tracking methods are those where the governing equations are solved separately for each fluid on both sides of the interface. The grid is quasifixed since it is only locally modified near the interface, to track the front separating both fluids $\left(\mathrm{Glimm}^{44}\right)$. More recently, interface-capturing methods have been developed where the interface is not defined as a boundary. Instead, the governing equations are solved on a fixed grid extending beyond the interface and the shape of the interface is determined by those grid cells where both fluids are present. One of such approaches is the marker-and-cell or MAC scheme (Harlow and $\mathrm{Welch}^{38}$ ) which follows particles of zero mass that are introduced initially in one of the fluids near the free surface. The most commonly used of the interface-capturing schemes might be the so-called volume-of-fluid (VOF, Hirt and Nichols ${ }^{45}$ ) and level set techniques (Sussman et al. $^{46}$ ). Chen et al. ${ }^{35}$ employed a piecewise linear version of the volume of fluid method to simulate breaking and splash up. The level set technique has been applied to predict overturning and breaking as well (Vogt and Kang, ${ }^{47}$ Vogt, ${ }^{48}$ and Iafrati and Campana ${ }^{36}$ ).

The numerical treatment of the interface in this study 


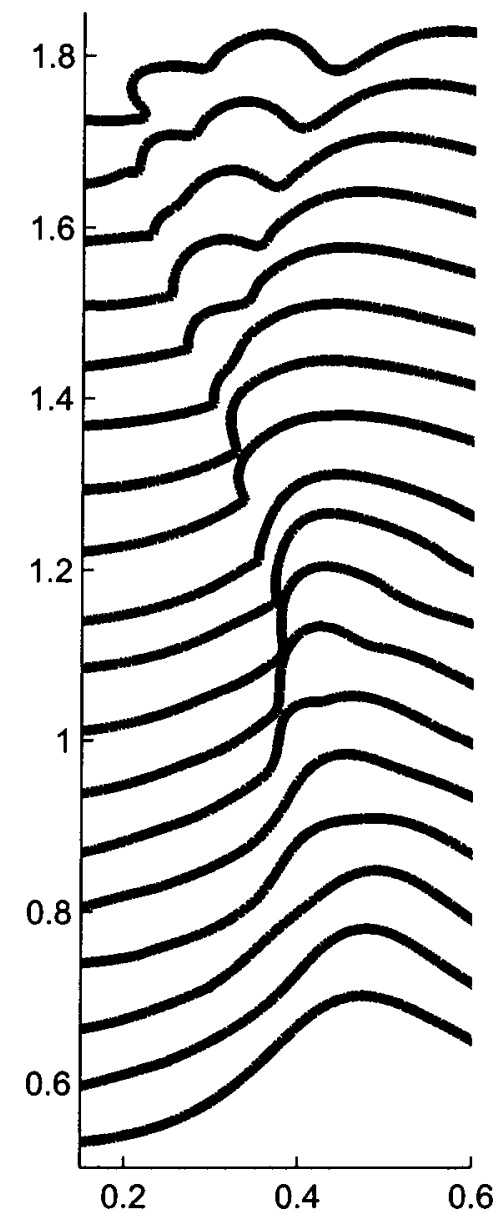

FIG. 6. Time sequence of free surface profiles corresponding to Fig. 4(c) $[\tau=0.0059,0.11183,0.1775,0.2195,0.2960,0.3552,0.4144,0.4736$, $0.5412,0.5803,0.6513,0.7105,0.7696,0.8288,0.8879,0.9471,1.0063$, 1.0653 (from bottom)]

makes use of a numerical technique that can be described as a hybrid between a front capturing and a front tracking technique (Unverdi and Tryggvason ${ }^{49}$ and Peskin ${ }^{50}$ ). It uses only one set of governing equations for the whole computational domain solved on a fixed grid, with a smooth density and viscosity variation through the deforming interface where the surface tension is computed. The transfer of front properties, such as the surface tension, to the fixed grid is done by ensuring that the quantity transferred is conserved. This is done via a two-dimensional interpolation consisting of area weighting such as that used by Tryggvason et al. ${ }^{51}$ Such interpolation requires values from two grid nodes in each direction. The treatment of the moving interface requires additional separate computational elements (i.e., line elements) to maintain accuracy while marking the interface which moves with the local velocity interpolated from the stationary grid. Such line elements are added or deleted in this study after every iteration to ensure that the size of each element is between 0.2 and 0.8 times the grid size. In this way the distribution of points, defining the line elements, on the interface can be controlled such that adequate resolution is maintained. In this study all computations have a minimum of 1024 line elements in the interface, however depending on the roughness of the interface as the flow evolves, the num- ber of line elements will change throughout the computation. These new elements are used to construct the density and viscosity fields, as indicated earlier, as well as to incorporate the surface tension effects. One of the advantages of this numerical treatment is that it does not require interpolations to locate the interface. This method has been used to examine various multiphase problems involving the rising and collision of bubbles and drop breakup and formation (i.e., Esmaeeli and Tryggvason ${ }^{52}$ and Han and Tryggvason ${ }^{53}$ ).

\section{Problem formulation}

The simulation of breaking waves propagating in the interface separating two different fluids is done in a rectangular computational domain as depicted in Fig. 1, where the corresponding density and viscosity for the bottom and top fluids are $\rho_{b}, \mu_{b}$ and $\rho_{t}, \mu_{t}$, respectively. Longuet-Higgins and Cocklet, ${ }^{54}$ Tanaka, ${ }^{55}$ and Jillians, ${ }^{56}$ among others, have worked on the form of the first superharmonic instability of periodic two-dimensional Stokes waves in a deep domain. It has been shown that the nonlinear development of the corresponding unstable modes may lead to breakup in the periodic regime.

This represents a simple condition to induce breaking in numerical simulations. In this study, the initial wave profile $\eta_{0}(x)$, horizontal velocity component $u_{0}(x, y)$, and vertical velocity component $v_{0}(x, y)$ correspond to a Stokes wave calculated at the second order of wave amplitude for an infinite domain (Dean and Darlymple ${ }^{57}$ ). The corresponding expressions are shown in the following equations:

$$
\begin{aligned}
\eta_{0}(x)= & \varsigma \cos (\kappa x)+\frac{a^{2}}{4} \frac{\cosh (\kappa h)}{\sinh ^{3}(\kappa h)}(2 \\
& +\cosh (2 \kappa h)) \cos (2 \kappa x),
\end{aligned}
$$

$$
\begin{aligned}
& u_{0}(x, y)= a \frac{g \kappa}{\beta} \frac{\cosh (\kappa y)}{\cosh (\kappa h)} \cos (\kappa x) \\
&+\frac{3}{4} \frac{a^{2} \beta \kappa \cosh (2 \kappa y)}{\sinh ^{4}(\kappa h)} \cos (2 \kappa x), \\
& v_{0}(x, y)= a \frac{g \kappa}{\beta} \frac{\sinh (\kappa y)}{\cosh (\kappa h)} \sin (\kappa x) \\
&+\frac{3}{4} \frac{a^{2} \beta \kappa \sinh (2 \kappa y)}{\sinh ^{4}(\kappa h)} \sin (2 \kappa x), \\
& \mathrm{s}=\frac{2 \pi a}{\lambda}, \quad \kappa=\frac{2 \pi}{\lambda}, \quad \beta^{2}=g \kappa \tanh (\kappa h),
\end{aligned}
$$

where $\lambda$ is the wavelength, $\mathrm{s}$ is the initial wave slope, $a$ is the initial wave amplitude from the mean water surface, $\kappa$ represents the wave number, and $h$ is the mean depth of the initial wave.

In the square computational domain, shown in Fig. 1, periodic boundary conditions in the direction of wave propagation are applied for the right and left boundaries, following a similar implementation to that of Patankar et al. ${ }^{58}$ 


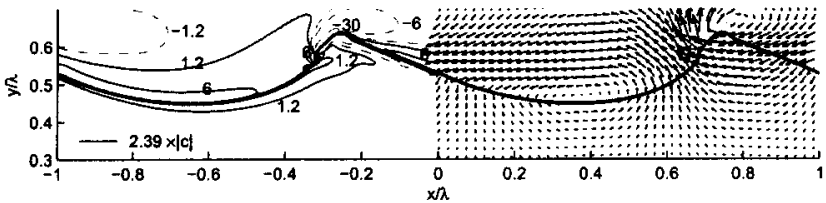

(a) $\tau=0.5969$

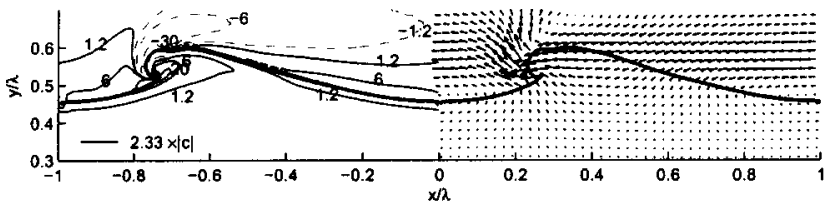

(c) $\tau=0.9365$

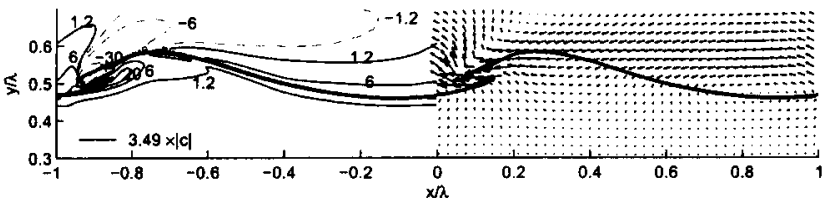

(e) $\tau=1.0301$

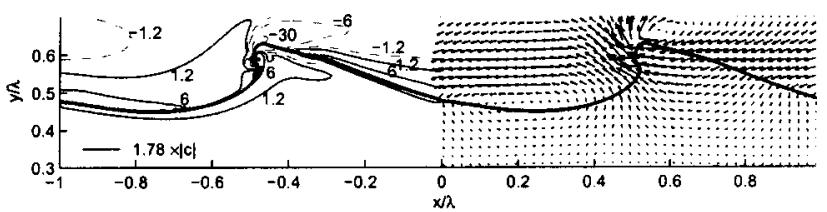

(b) $\tau=0.7258$

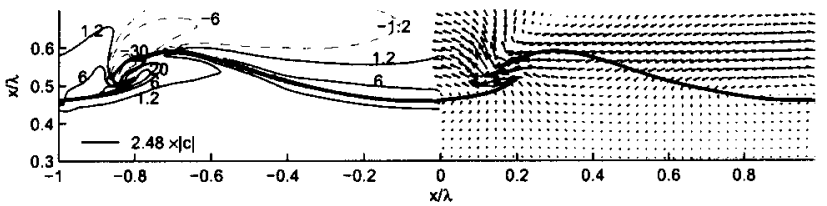

(d) $\tau=0.9892$

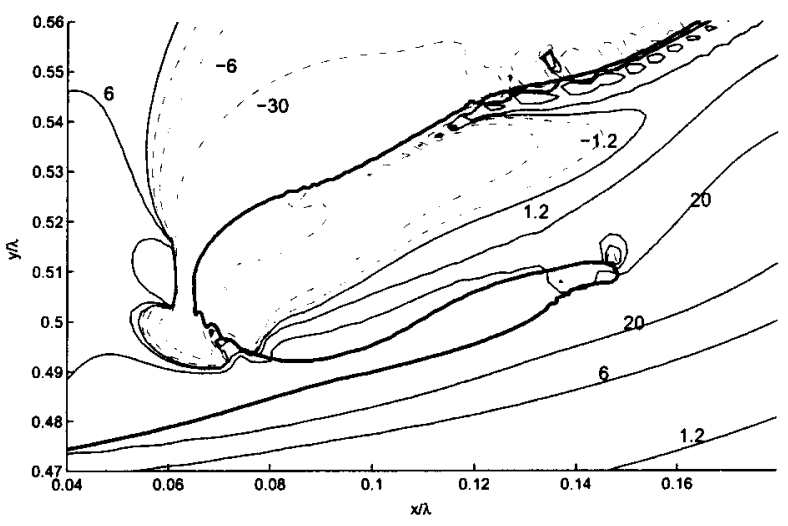

(f) $\tau=1.0301$

FIG. 7. Time sequence of free surface profiles showing the velocity field the vorticity contours $(B o=359, \operatorname{Re}=350$, density ratio $=0.028$, viscosity ratio $=0.5$, and $\varsigma=0.5507)$. Positive vorticity contour $\left(\omega^{+}\right)---$, negative vorticity contour $\left(\omega^{-}\right)---. \omega^{+}=20,6,1.2$ and $\omega^{-}=-30,-6,-1.2$ are plotted for subplots (a)-(e). Subplot (f) is a close-up view of subplot (e).

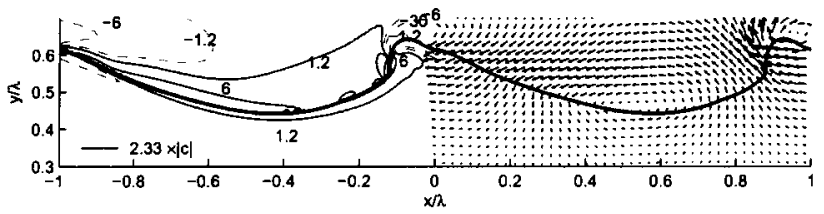

(a) $\tau=0.4202$

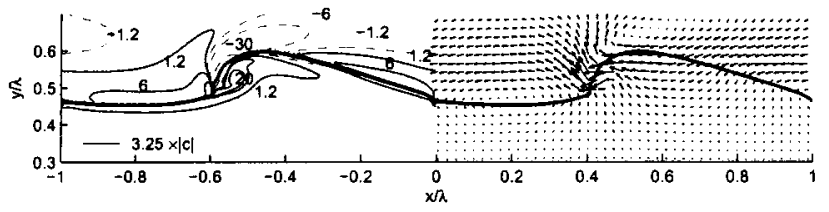

(c) $\tau=0.7577$

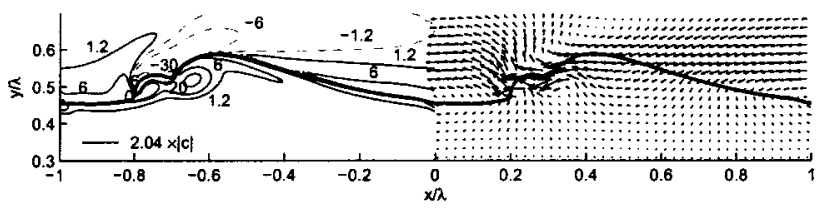

(e) $\tau=0.8876$

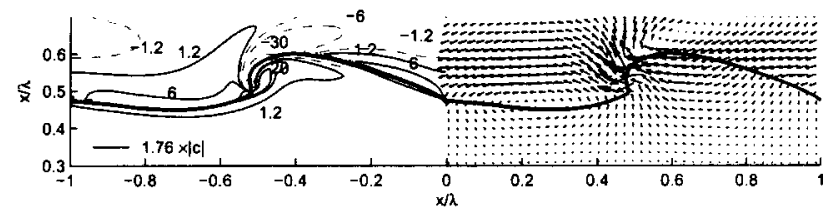

(b) $\tau=0.7103$

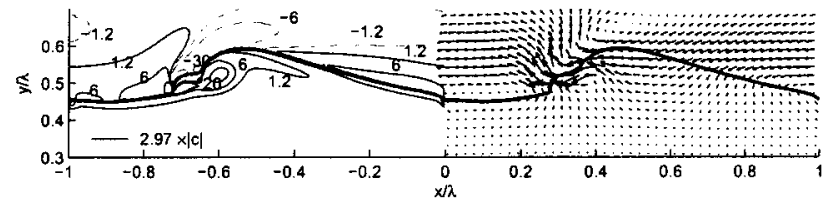

(d) $\tau=0.8344$

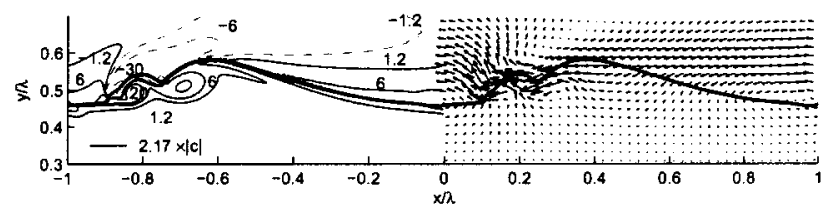

(f) $\tau=0.9469$

FIG. 8. Time sequence of free surface profiles showing the velocity field and the vorticity contours for $B o=38(\operatorname{Re}=350$, density ratio $=0.028$, viscosity ratio $=0.5$, and $\mathrm{s}=0.5507)$. 


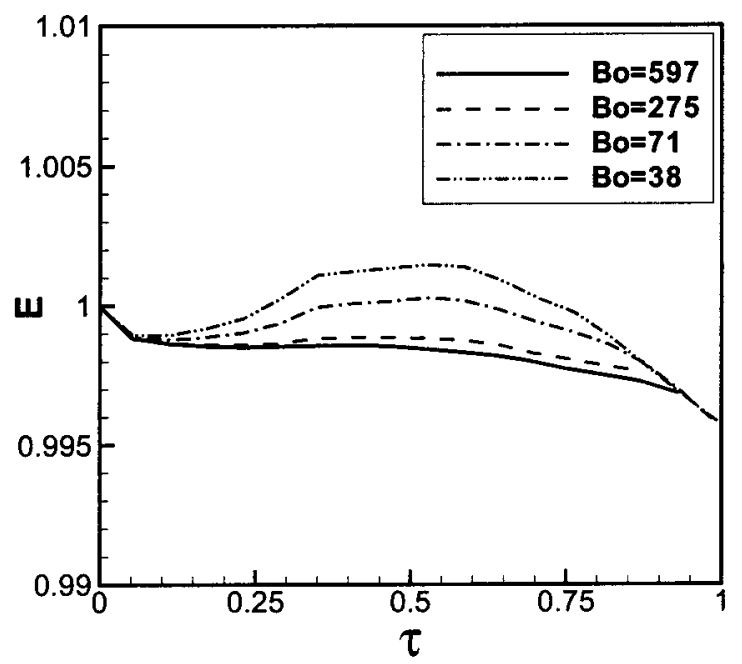

(a) Total Energy (E)

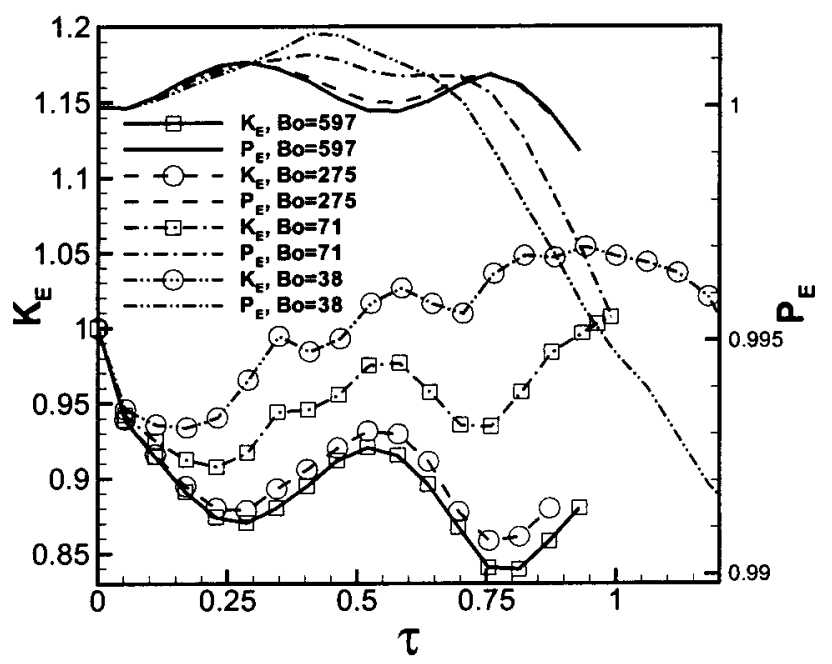

(b) Kinetic Energy $\left(K_{E}\right)$ and Potential Energy $\left(P_{E}\right)$

FIG. 9. Time evolutions of total wave energy and wave potential and kinetic energies as a function of Bond number $(\operatorname{Re}=350$, density ratio $=0.028$, viscosity ratio $=0.5$, and $s=0.5507$ ).

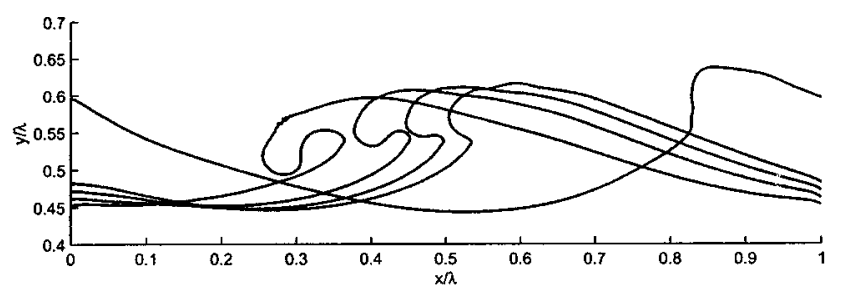

(a)

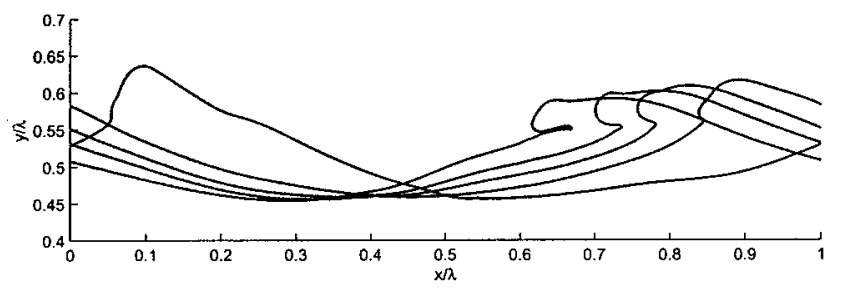

(b)

FIG. 10. Comparison of overall wave evolution $(\mathrm{Re}=350, \mathrm{Bo}=224$, density ratio $=0.028$, viscosity ratio $=0.028$ and $s=0.5654$ ) (a) second order, $\tau$ $=0.48645,0.72109,0.81202,0.89927$, (b) fifth order, $\tau=0.33452$, $0.48039,0.52401,0.56744,0.62846$.

$$
\begin{aligned}
& \phi(x, y)=\phi(x+\lambda, y)=\phi(x+2 \lambda, y)=\cdots, \\
& \phi=u, v, p, \eta, \rho, \mu .
\end{aligned}
$$

A full-slip boundary condition is applied along the bottom boundary and consequently the gradient of the velocity along the $x$ and $y$ directions is set to zero. An outflow condition is used for the top edge of the computational domain. The average position of the interface is midway between the top and bottom of the domain. This numerical study targets wave progressing in an infinite domain. The finite size of the computational domain was chosen to be large enough to have no influence on the computations, and it is one wavelength in both the streamwise and normal directions.

The wave breaking problem is defined by four nondimensional parameters, those are the bottom layer Bond and Reynolds numbers and the density and viscosity ratios be-

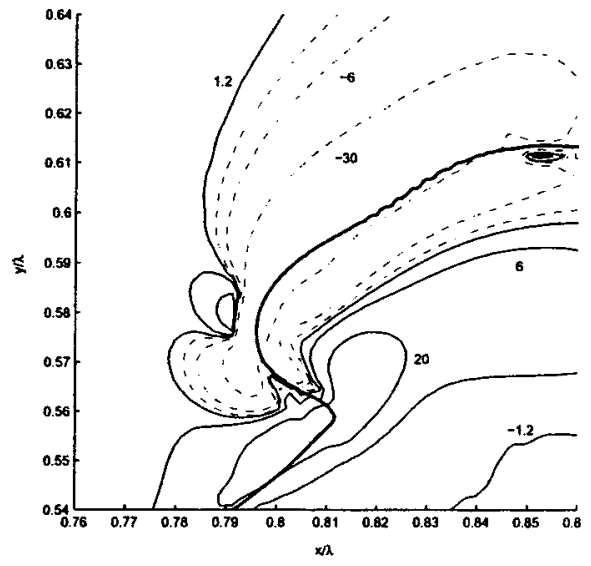

(a)

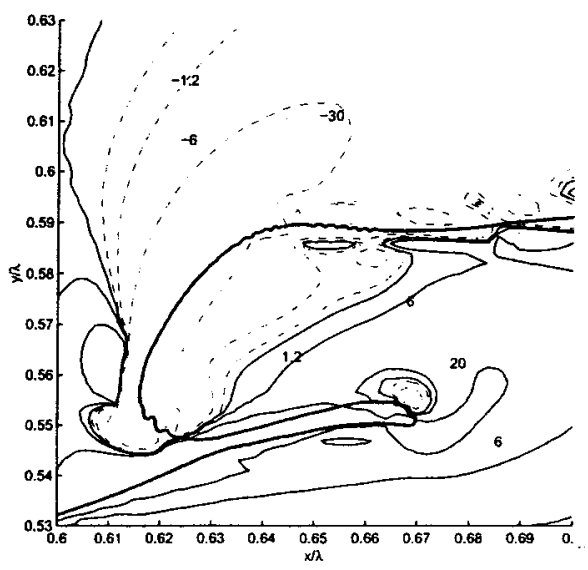

(b)
FIG. 11. Vorticity contours $(\mathrm{Re}=350$, $\mathrm{Bo}=224$, density ratio $=0.028$, viscosity ratio $=0.3$, and $s=0.5654$ ) for wave simulations with fifth order Stokes wave as initial condition (a) $\tau$ $=0.48422$, (b) $\tau=0.62846$. 


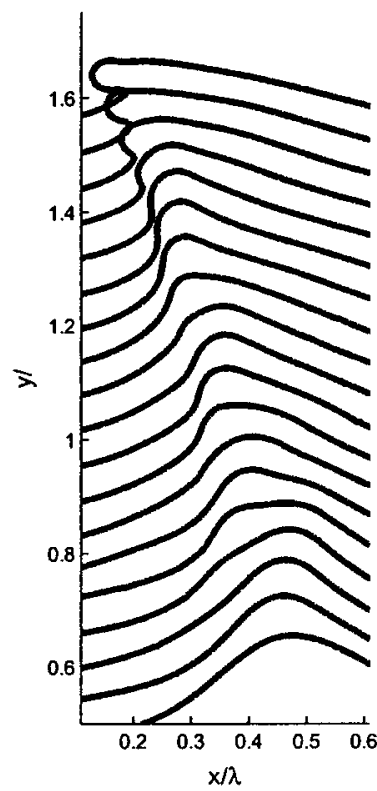

(a) $R e=175$

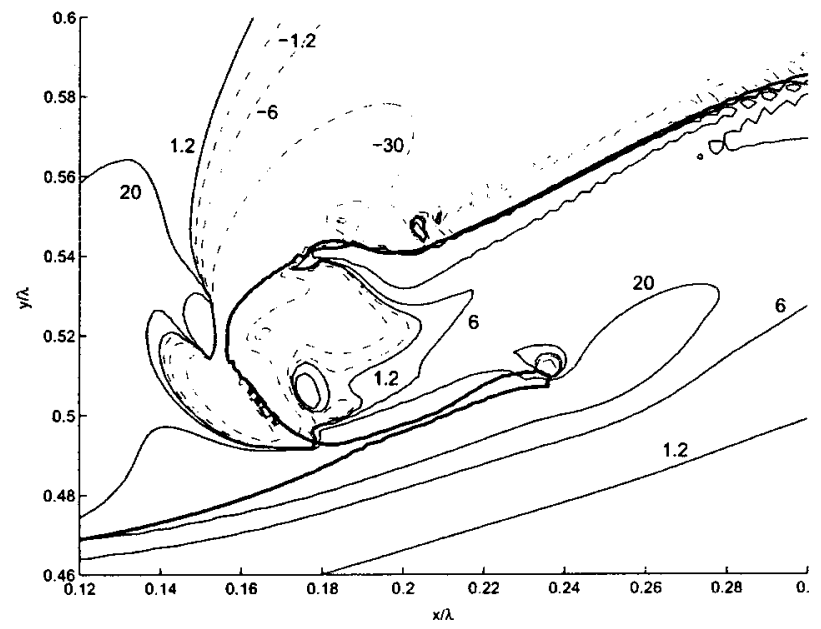

(c) vorticity distribution

FIG. 12. Time sequence of free surface profiles for different Reynolds number, $\mathrm{Re}=175,500(\mathrm{Bo}=224$, density ratio $=0.028$, viscosity ratio $=0.5$, and $\mathrm{s}=0.5507$ ). (a) $\mathrm{Re}=175, \tau=0.02926,0.0878,0.1464,0.2038,0.2635$, $0.3192,0.3778,0.4363,0.4949,0.5535,0.6120,0.6705,0.7291,0.7888$, $0.8462,0.9048,0.9634,1.0220,1.0816$ (from bottom). (b) $\operatorname{Re}=500, \tau$ $=0.0084,0.1673,0.2509,0.3346,0.4176,0.4751,0.5455,0.5911,0.6268$, $0.6465,0.6971,0.7800,0.8637,0.9263,0.9569$ (from bottom). (c) Vorticity distribution $(\operatorname{Re}=500, \tau=9569)$.

tween the top and bottom fluids, since the corresponding physical process depends on the fluid inertia, viscous, surface tension, and gravity forces. These parameters are defined as

$$
\begin{aligned}
& \mathrm{Bo}=\frac{\rho_{b} g}{\sigma \kappa^{2}}, \quad \operatorname{Re}=\frac{c \rho_{b}}{\mu_{b} \kappa}, \\
& \hat{\rho}=\frac{\rho_{t}}{\rho_{b}}, \quad \hat{\mu}=\frac{\mu_{t}}{\mu_{b}},
\end{aligned}
$$

where $c$ is the phase velocity based on the linear dispersion

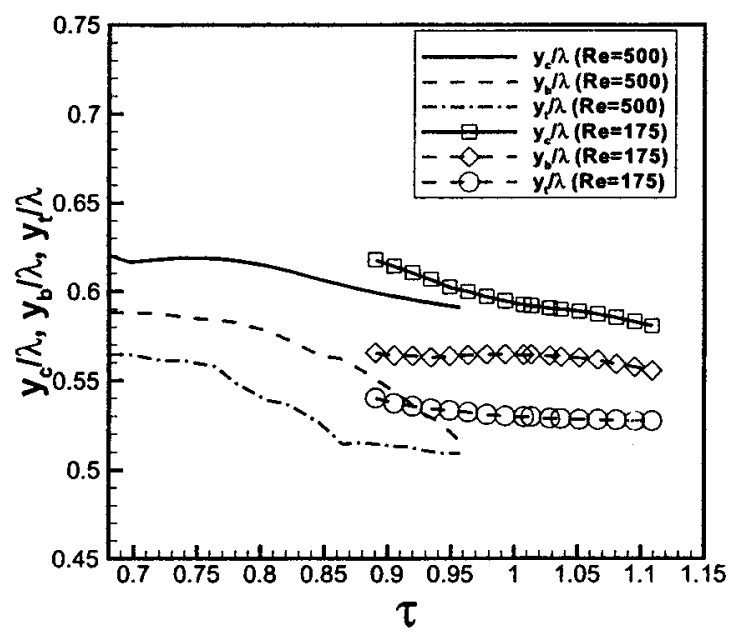

(a) $y_{c}, y_{b}, y_{t}$

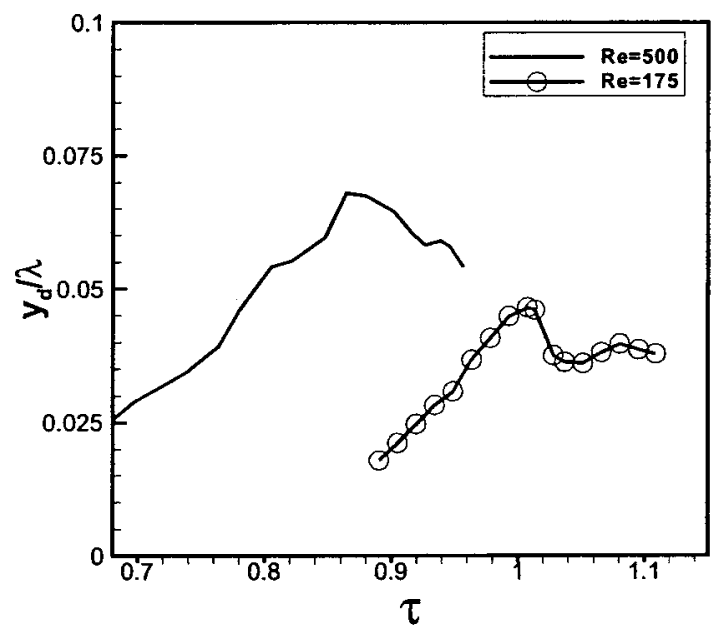

(b) $y_{d}$

FIG. 13. Geometrical parameters of wave profile as a function of Reynolds number, $\mathrm{Re}=175,500 \quad(\mathrm{Bo}=224$, density ratio $=0.028$, viscosity ratio $=0.5$, and $\mathrm{s}=0.5507)$.

relation. In this study, length, velocity components, and time are normalized by wavelength $\lambda$, phase velocity $c$, and $\lambda / c$, respectively.

The initial wave amplitude or wave steepness (defined as the ratio of the wave amplitude to the wavelength) influences the wave motion, to the point of being critical for breakup to take place. The influence of this parameter on the flow evolution is also discussed in this study.

\section{RESULTS AND DISCUSSIONS}

\section{A. Grid refinement test}

Grid refinement tests were carried out to determine the adequate numerical resolution for the problem at hand. Three grids of resolutions $480 \times 480$ (coarse), $512 \times 512$ (medium), and $544 \times 544$ (fine) were tested and the evolution of the interface for each grid is shown in Fig. 2 at $\tau=0.8197$ for $\mathrm{Re}=350, \mathrm{Bo}=224, \mathrm{~s}=0.5654$ and density and viscosity ra- 


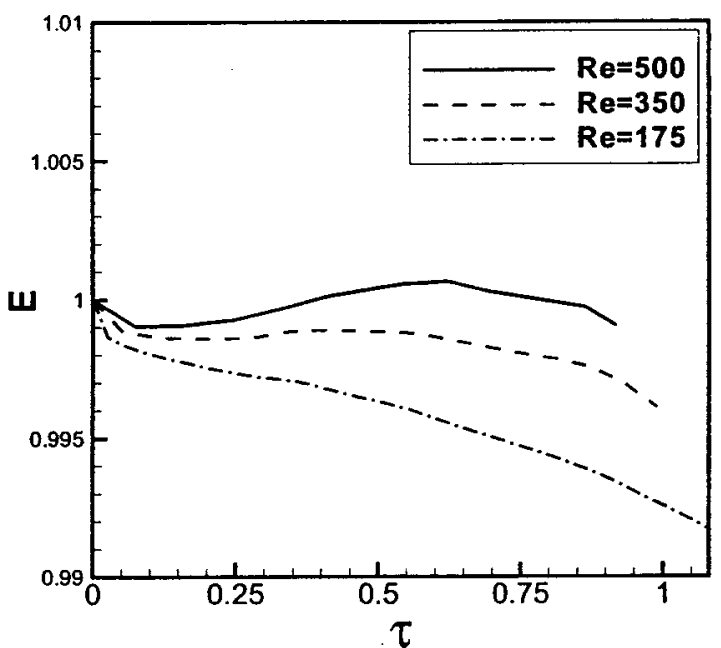

(a) Total Energy (E)

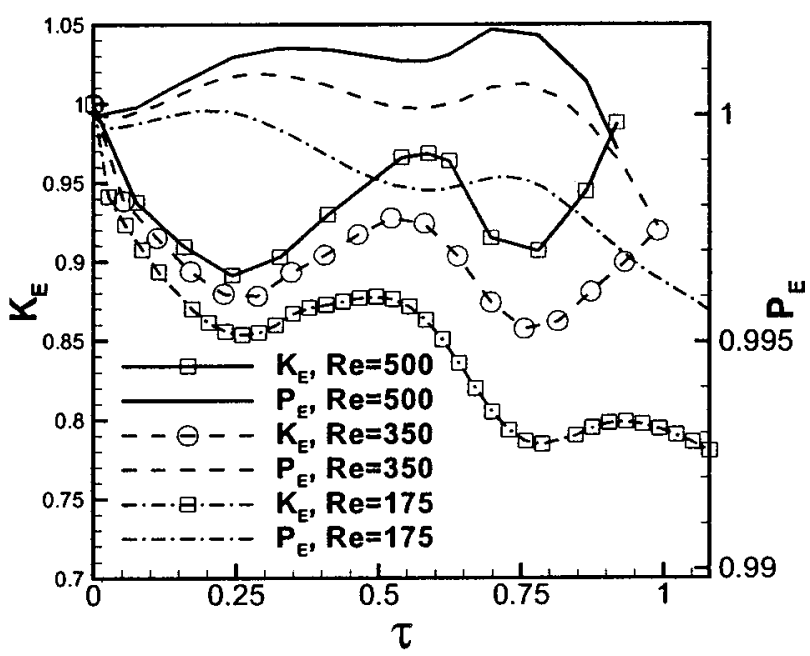

(b) Kinetic Energy $\left(K_{E}\right)$ and Potential Energy $\left(P_{E}\right)$

FIG. 14. Time evolution of total wave energy and wave potential and kinetic energies as a function of Reynolds number $(\mathrm{Bo}=224$, density ratio $=0.028$, viscosity ratio $=0.5$, and $\varsigma=0.5507$ ).

tios of 0.028 and 0.3 , respectively. Better resolved and very similar interfaces were found for the medium and fine grids compared to the coarse grid. The results presented in this paper correspond to grids with $512 \times 512$ resolution to reduce the computational time, without compromising accuracy for the range of parameters of interest. All computations have a minimum of 1024 line elements defining the interface. The number of elements is recomputed after every iteration as explained in Sec. II B. For the example shown in Fig. 2 the final number of line elements defining the interface at the end of the computation was 1446.

\section{B. Description of general plunger breaking}

The evolution of breaking waves is studied here for a range of values of each of the governing nondimensional parameters. This range is based on the computational re- sources available and the performance of the computational methodology employed. Such limitations are not uncommon and can be found in a number of numerical simulations of water waves, such as for example the study of the dissipation of surface waves by Yang and Tryggvason ${ }^{59}$ and the study of breaking waves by Chen et al. ${ }^{33}$ The former study numerically computes finite amplitude water surface waves with density ratios of $0.01,0.02$, and 0.04 and a kinematic viscosity ratio of 1 and shows a good agreement with viscous linear theory of water waves. The work of Chen et al. ${ }^{33}$ simulates a breaking wave for a density ratio of 0.01 and viscosity ratio of 0.4 and again the results are qualitatively very similar to those observed in experimental studies. The parameters used in this study are not representative of realistic water waves. Nonetheless, the work attempts to explain the influence of each of the governing nondimensional parameters in the mechanism of breaking. As the numerical values of the parameters get closer to those corresponding to water waves it is expected that the breakers found will be qualitatively similar to water breaking waves. The study is also relevant to the understanding of the physical processes involved on the breaking of internal waves.

Deep water gravity-capillary waves with wavelengths in the interval $(0.004 \mathrm{~m}, 0.07 \mathrm{~m})$, have corresponding Reynolds numbers of 211 and 3681, and Bond numbers 0.054 and 16.4 , respectively. In this study a plunging breaking wave is initially studied for a chosen set of nondimensional parameters. The surface tension effects are then analyzed by studying the effects of the Bond number as it varies from 38 to 597. The changes are achieved by modifying the surface tension coefficient of the interface by choosing the gravitational acceleration from the linear dispersion relation after setting the phase speed to unity. The Reynolds number effects are then studied by varying Re from 175 to 500 . Such range is accomplished by changing the viscosity corresponding to the bottom fluid. Modifications of the density ratio and viscosity ratio are obtained by adjusting the density and viscosity corresponding to the top fluid, while the density of the lower fluid is set to 1 and the viscosity is adjusted based on the Reynolds number. The range for the density ratio is within $(0.02,0.2)$ and the range of the viscosity ratio is within $(0.3$, $0.7)$. Finally the effects of the wave slope are also studied in the range $0.5507 \leqslant \varsigma \leqslant 0.5654$.

Figure 3 shows a time series of the evolution of the plunging wave corresponding to the following parameters: $\mathrm{Re}=350, \mathrm{Bo}=224, \mathrm{~s}=0.5581$ and density and viscosity ratios of 0.02 and 0.5 , respectively. Each plot shows the instantaneous vorticity field in the left part of the figure, and the velocity vectors, shown every 12 grid nodes, in the right part of the figure. The initial wave moves from right to left. In the early stages of wave motion, movement of the top fluid is induced through the dynamic coupling at the interface, and maximum vorticity is found at the crest [Fig. 3(a)]. As the flow evolves, the wave front becomes steeper until it eventually becomes vertical [Fig. 3(b)]. As the wave front becomes steeper the wave flow seems to converge toward the front face [Fig. 3(b)]. The maximum velocity in this case is 2.591 with a corresponding horizontal component of 


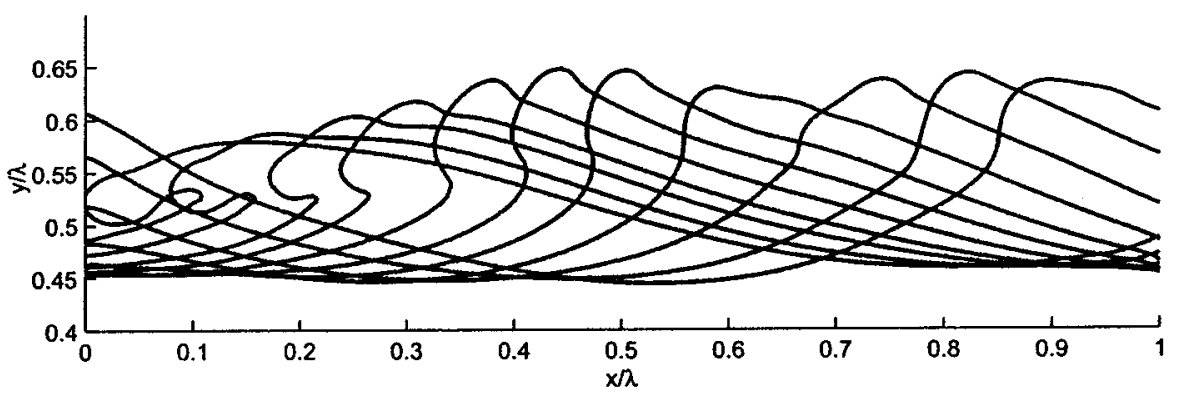

(a) $\hat{\rho}=0.04, \tau=0.4684,0.5354,0.6191,0.7027,0.7780,0.8366,0.8952,0.9537,0.9945$, 1.1060 (from right).

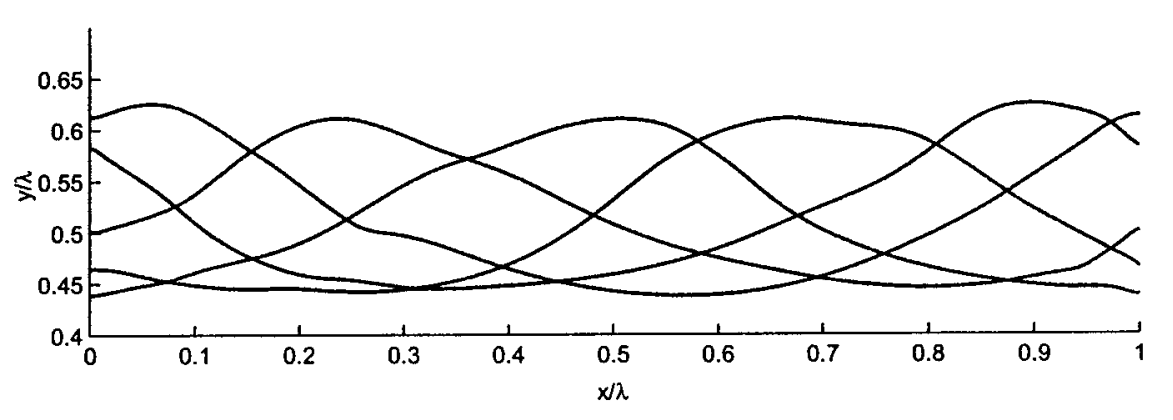

FIG. 15. Time sequence of free surface profiles for density ratios density ratio $=0.04$ and $0.2 \quad(\operatorname{Re}=350, B o$ $=224$, viscosity ratio $=0.5$, and $\mathrm{s}$ $=0.5581)$. Note: Fig. 3 already showed the time sequence of free surface profiles for density ratio $=0.02$ $(\mathrm{Re}=350, \quad \mathrm{Bo}=224$, density ratio $=0.02$, viscosity ratio $=0.5$, and $\varsigma=0.5581)$.

(b) $\hat{\rho}=0.2, \tau=0.5015,0.6689,0.8780,1.1291,1.3383$ (from right).

-1.172 . These large velocities take place at the top of the wave crest. The vorticity distribution shown in those plots indicates that the peak vorticity in the top fluid follows the wave front and the vorticity originally concentrated at the wave crest is subsequently shed. The front face of the crest develops into an overturning liquid jet with a rounded end. In the process of jet formation [Figs. 3(c)-3(e)] a small kink, bulge, is formed at the point in the wave front where the wave slope changes signs [Fig. 3(d)]. That singular point comes associated with a discontinuity in tangential velocity across the interface, which in turns translates, into vorticity as shown in Figs. 3(d) and 3(e). The kink, which constitutes the jet tip, then gets progressively enlarged and rounded toward the wave crest [Figs. 3(d)-3(e)]. The jet once formed increases its length until it eventually touches down on the forward face of the wave [Fig. 3(f)]. Vorticity is mostly concentrated close to the interface and increases progressively with jet formation and flow overturning. The vorticity contours in the lower fluid show that the jet formation is associated with vorticity being shed downstream. The rest of the flow away from the thin layer at the interface and the jet is essentially irrotational. The jet formation and consequent enlargement comes associated with very large velocities in the jet region. The maximum velocity evolves from 1.805 , with a corresponding -1.155 horizontal component in Fig. 3(c), to 2.072, with a -1.375 horizontal component in Fig. 3(e). The largest velocity is located at the wave crest and the uppermost part of the wave front in Fig. 3(d), and in the prominent jet in Fig. 3(e). The horizontal velocity is a critical parameter in jet formation and overturning. It is clear from the latter that the largest horizontal particle speeds are much greater than the phase velocity. Figure 3(f) shows a clear engross- ment of the jet tip as it approaches the forward face of the wave in anticipation to splash. The splash up process that takes place after jet impingement is not shown in this study. The overall process of wave evolution and breaking presented in Fig. 3 seems to agree qualitatively with the numerical results of Chen et al., ${ }^{35}$ the experimental results of Bonmarin, ${ }^{11}$ and the flow visualizations of Perlin et al. ${ }^{14}$ The numerical results, however, do not capture any parasitic capillary waves along the lower front face of the plunger as observed by the flow visualizations of Perlin et al. ${ }^{14}$

\section{Surface tension effects on wave breaking}

The Bond number is a measure of the relative strength of the buoyancy and surface tension forces. To study the effect of the surface tension on wave breaking, numerical experiments are conducted by systematically changing the Bond number while maintaining the other nondimensional parameters constant with an initial wave steepness of 0.5507 . In doing so, the changes induced by the surface tension on the process that results on wave breaking can be assessed.

Figures 4(a)-4(c) show the free surface evolution corresponding to three different simulations with Bond numbers of 597, 275, and 38, respectively. Simulations were run also for Bond numbers 359 and 71 , but the results were very similar to those presented for $\mathrm{Bo}=597$ and 38 , respectively. The main differences are that the larger the Bond number, the faster the evolution of the breaker, and the steeper it becomes. Consequently the increasing role of surface tension comes associated with a significant reduction in jet intensity and air entrapment. While the jet formation and overturning are clearly seen in Figs. 4(a) and 4(b), in Fig. 4(c) a sharp 


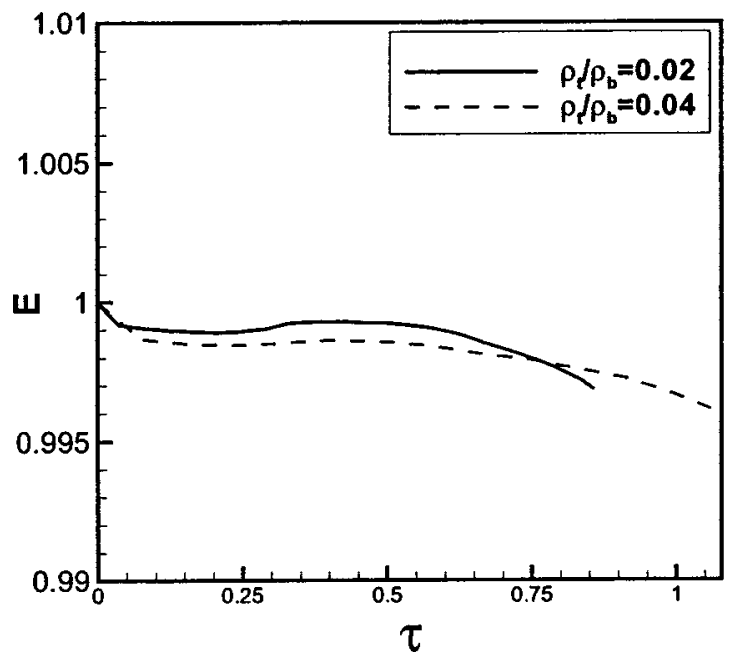

(a) Total Energy (E)

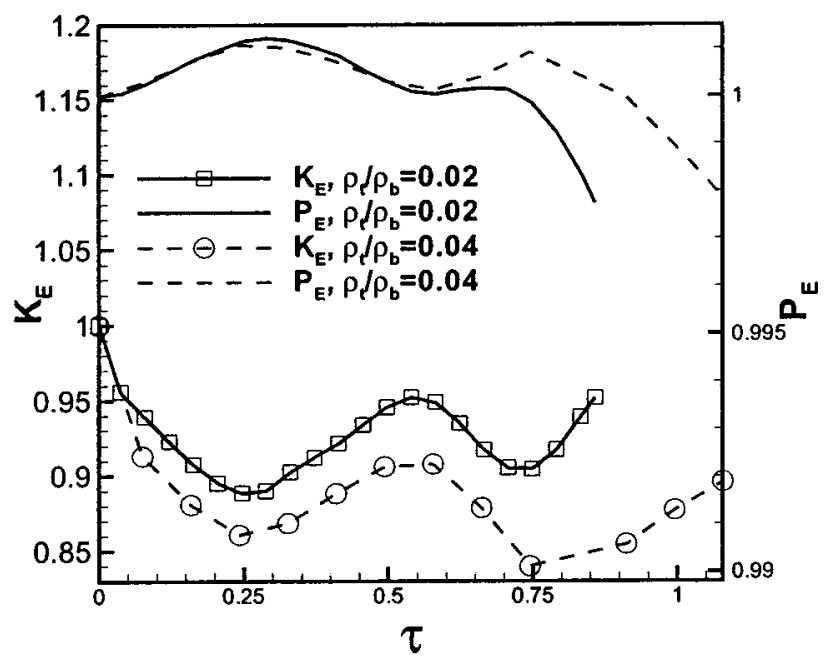

(b) Kinetic Energy $\left(K_{E}\right)$ and Potential Energy $\left(P_{E}\right)$

FIG. 16. Time evolutions of total wave energy and potential and kinetic energies as a function of density ratio $(B o=224$, density ratio $=0.028$, viscosity ratio $=0.5$, and $s=0.5581$ ).

corner develops at the bottom of the leading edge and the jet is completely suppressed. The large curvature present at the jet root is responsible for the development of a strong shear layer that seems to contribute to the presence of parasitic waves in the cases presented in Figs. 4(a) and 4(b), while they do not seem to be present for the Bond number case shown in Fig. 4(c). Their presence was never detected prior to overturning and only one or two of such small amplitude waves were seen. They seem to have some similarities with the well-known parasitic capillary waves. The potential flow calculations of Longuet-Higgins ${ }^{31}$ and Ceniceros and $\mathrm{Hou}^{29}$ have shown the appearance of capillary waves in front of the toe for water waves. Longuet-Higgins ${ }^{31}$ argues that the vorticity produced by the capillary waves might be responsible for the appearance of longer capillary ripples above the toe that propagate downstream. Such ripples have also been ob- served experimentally by Duncan et al. ${ }^{19}$ who concluded that they appear after the toe begins to move. Duncan and Dimas $^{60}$ argue, based on linear stability analysis of a measured velocity profile, that the ripples are generated by the instability of a shear layer produced between "a gravityinduced downslope flow near the surface and the underlying upslope flow." Downstream propagating ripples are observed in the present simulations for the case shown in Fig. 4(c). The ripples seem to change amplitude and wavelength as they propagate. The presence of ripples in that case does not come associated however with parasitic capillary waves.

In all cases presented in Fig. 4 it can be seen that a bulge appears in the front of the wave as it becomes steeper. Some geometrical parameters representative of the evolution of the waves, such as the crest and toe heights (all heights are defined with respect to the bottom of the computational domain, see Fig. 1) and the bulge thickness (determined as the distance between the line defined by the crest and the toe and the point of the bulge with tangent parallel to the latter line), are defined and presented in Fig. 5(a). The evolution of each of these parameters, for the various Bond numbers shown in Fig. 4, along with the evolution of the amplitude and wavelength of the first ripple obtained for $\mathrm{Bo}=38$ is shown in Figs. 5(b)-5(d). The computations of these geometrical parameters were done at only a few time steps of the computed solutions.

Figure 5(b) shows the evolution of the amplitude and wavelength corresponding to the first ripple computed for $\mathrm{Bo}=38$. Both its amplitude and wavelength seem to consistently increase in time except during $0.94<\tau<0.98$, which corresponds to the time when the second ripple appears as shown in Fig. 6 and discussed later in this section. Figure 5(c) shows that the changes in the crest height are initially small for all Bond numbers. A maximum of $0.064 \lambda$ and $0.063 \lambda$ take place for $\mathrm{Bo}=597$ and 275 , respectively, at approximately the same time $\tau=0.78$. The crest height decreases for both Bo to approximately the same value, $0.06 \lambda$, at $\tau=0.95$ with an almost identical subsequent evolution. The height of the toe is initially almost the same for the waves with $\mathrm{Bo}=597$ and 275 and evolves similarly thereafter up to $\tau=0.78$. This time coincides with the time when the maximum crest height takes place and the time when ripples begin to appear as shown in Fig. 6. The decrease of the toe height is faster after this point until approximately $\tau=0.95$. Afterwards $y_{t}$ remains almost constant for $\mathrm{Bo}=597$ while it continues to decrease at the same decay rate for $\mathrm{Bo}=275$. The height of the bulge is very similar for both $\mathrm{Bo}=597$ and 275 up to approximately $\tau=0.95$, after that their rate of decay differ being slightly faster for the lower Bo. The evolution of the thickness of the bulge represented in Fig. 5(d) for $\mathrm{Bo}=597$ and 275 shows a constant increase up to approximately $\tau=0.78$ when the maximum crest height is reached. The decay of the crest and toe heights corresponding to the simulation for $\mathrm{Bo}=38$ is also shown in Fig. 5(c). The rate of decay is comparatively much slower with respect to the results shown for the higher Bond numbers.

The toe seems to move downwards as the bulge slides down in the simulation corresponding to the lowest Bond number 38. The motion of the toe can be appreciated in Fig. 


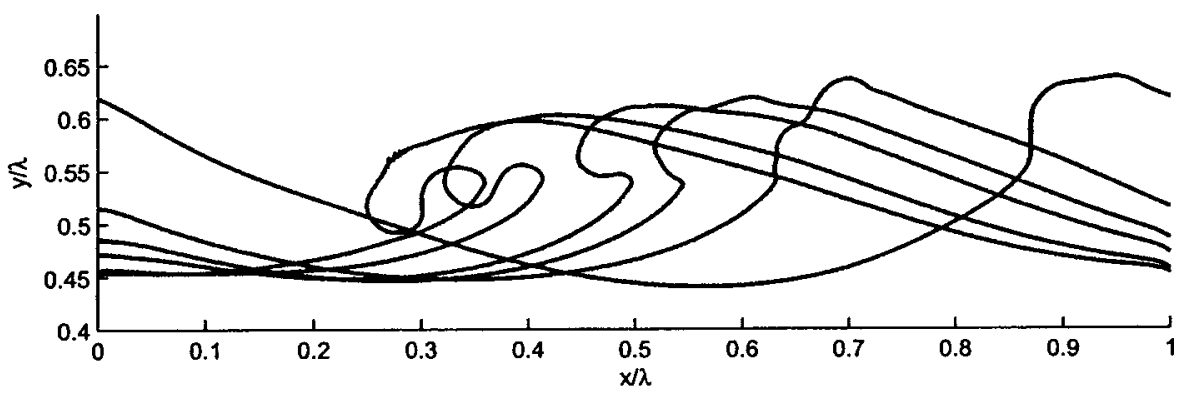

(a) $\hat{\mu}=0.3, \tau=0.4326,0.6186,0.6863,0.7351,0.8260,0.8730$ (from right).

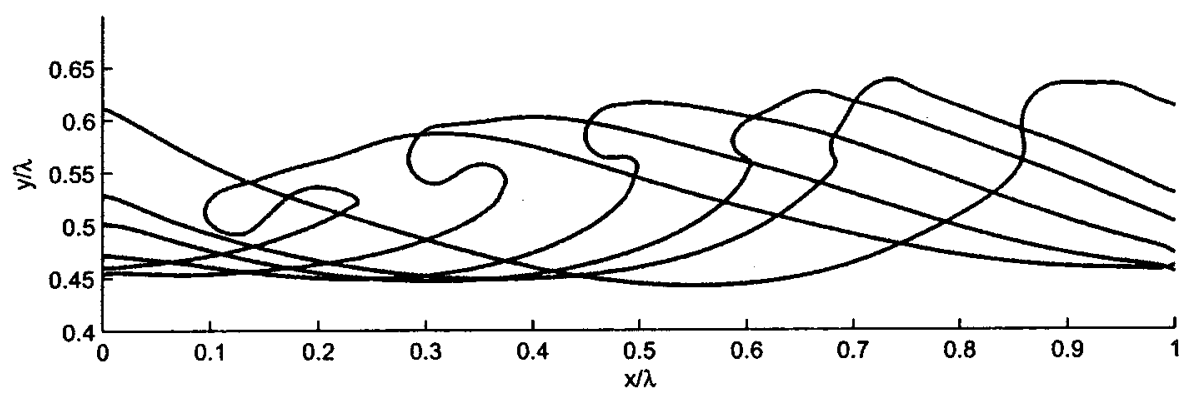

(b) $\hat{\mu}=0.5, \tau=0.4446,0.5851,0.6436,0.7372,0.8527,0.9713$ (from right).

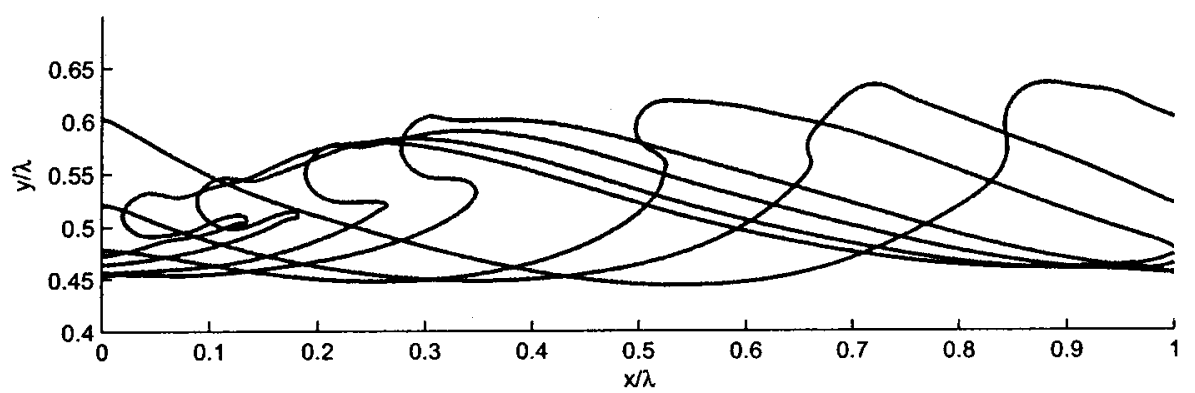

(c) $\hat{\mu}=0.7, \tau=0.4602,0.6018,0.7105,0.8735,0.9362,0.9742,1.0365$ (from right).

6 , where a profile history displayed in a coordinate system fixed with respect to the wave crest is shown. For clarity each profile is displaced vertically a distance corresponding to $(\Delta \tau \times c)$, where $\Delta \tau$ corresponds to the time between two neighboring profiles and is indicated in the label of Fig. 6. The bulge starts to develop around $\tau=0.3552$. The position of the toe remains fairly stationary with respect to the crest while the bulge grows in amplitude. Around $\tau=0.5412$ the toe starts to slide down the front of the wave and the first ripple appears between the toe and the crest around $\tau$ $=0.75$. The second ripple appears around $\tau=0.94$. The ripples travel at a lower speed than the wave phase speed, but their amplitude and wavelength seem to increase as they go. Also noticeable from the figure is the movement of the toe before the appearance of the ripples. The toe seems to quickly reach a maximum distance from the crest at $\tau=1$ and then seems to remain unchanged up to the end of the computations. These results are consistent with the experimental data of Duncan et al. ${ }^{20}$

Figures 7(a)-7(e) show the details corresponding to the vorticity distribution and velocity vectors for different times
FIG. 17. Time sequence of free surface profiles for viscosity ratios viscosity ratio $=0.3,0.5$, and $0.7(\mathrm{Re}$ $=350, \mathrm{Bo}=224$, density ratio $=0.028$, and $\mathrm{s}=0.5654)$. throughout the evolution of the breaker corresponding to $\mathrm{Bo}=359$ with the same other nondimensional parameters as those shown in Fig. 4. The evolution of the breakers corresponding to $\mathrm{Bo}=359$ and 597 are very similar. Figure 7(f) shows an enlarged view of the vorticity distribution corresponding to Fig. 7(e). The formation of the bulge in the front of the wave can be seen in Fig. 7(a), where the flow is shown to converge toward it. In the same figure the early stages of the formation of a shear layer in the bulge can be seen. The maximum velocity is 2.39 with a corresponding horizontal component of -1.322 and it is located at the bulge. The maximum velocity corresponding to Fig. 7(c) prior to the plunging of the jet is 2.3341 , is located at the bulge and has a corresponding value of -2.3083 for the horizontal component of the velocity. The corresponding maximum horizontal component of the velocity for Fig. 7(e) is -1.6487 . As expected the horizontal velocity component reaches a maximum absolute value prior to plunging and decreases afterwards. The vorticity generated at the toe is shed downstream and eventually gets to interact with the free surface as shown in Figs. 7(c)-7(e). In Fig. 7(f) the trace of a small amplitude, 


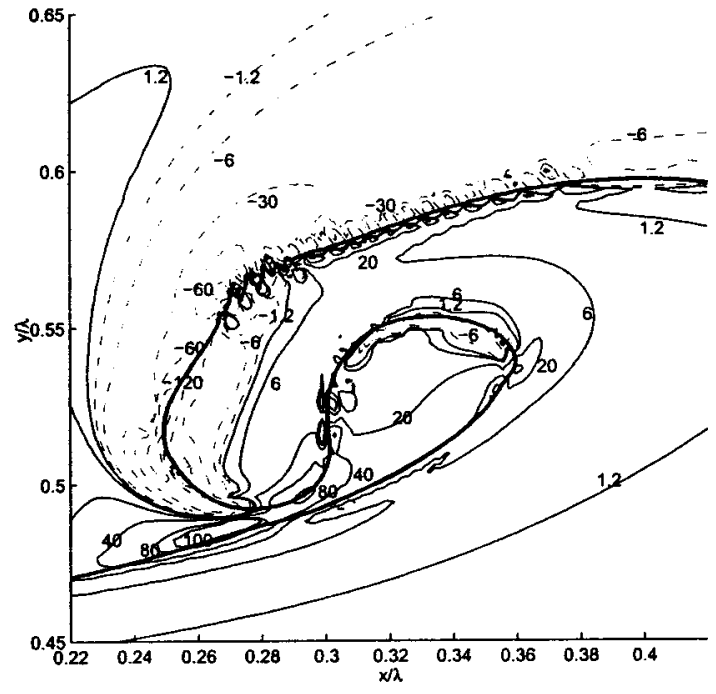

(a) $\hat{\mu}=0.3, \tau=0.8730$

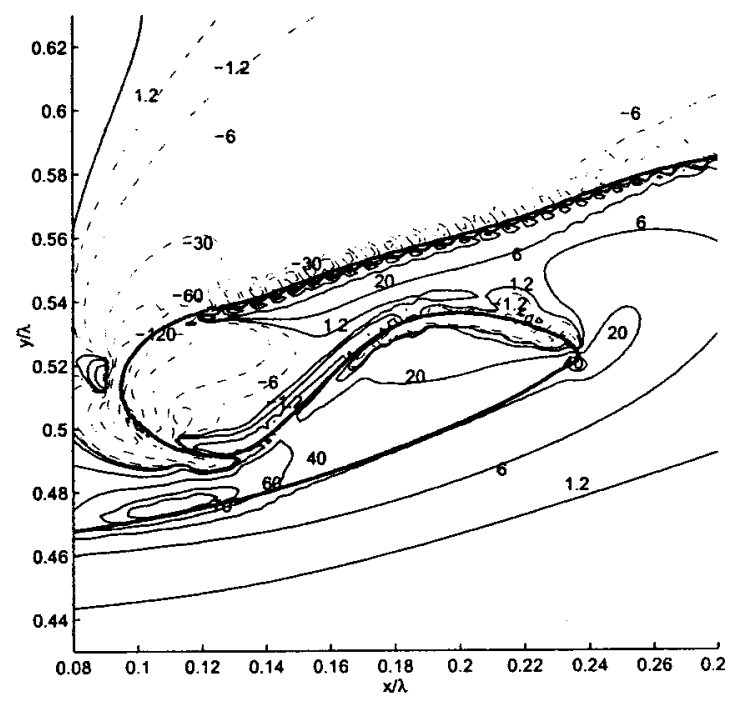

(b) $\hat{\mu}=0.5, \tau=0.9713$

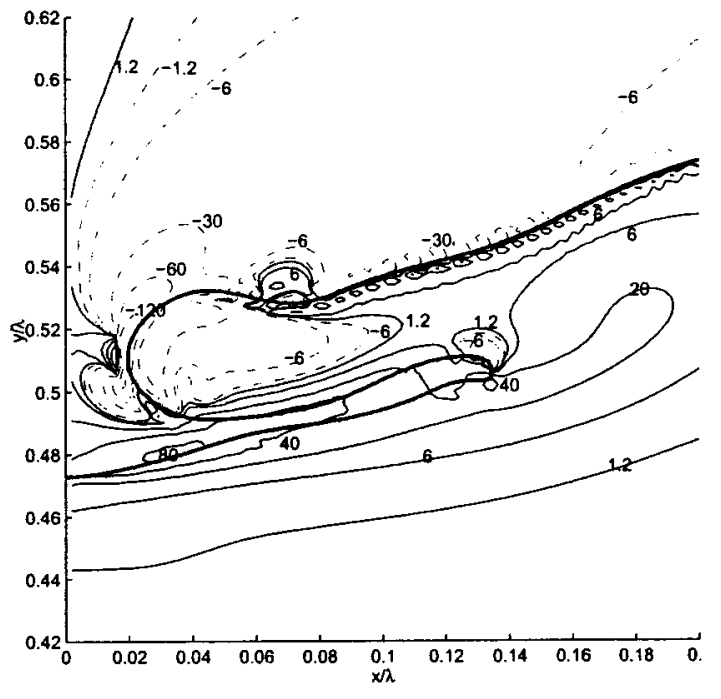

(c) $\hat{\mu}=0.7, \tau=1.0365$

FIG. 18. Comparisons of vorticity contours prior to the impingement of the jet for different viscosity ratios $(\operatorname{Re}=350, B o=224$, density ratio $=0.028$, and $\varsigma=0.5654$ ). (a) Viscosity ratio $=0.3, \tau=0.8730$, (b) viscosity ratio $=0.5, \tau=0.9713$, (c) viscosity ratio $=0.7, \tau=1.0365$.

short-lived ripple can be seen on the upper portion of the front as a result of the instability of the shear layer in the bulge. In the same figure the trace of a parasitic capillary wave close to the toe can also be appreciated. In the simulations corresponding to the larger Bond numbers one or two such waves were also appreciable, however their amplitude was always very small and they did not seem to appear prior to overturning. The lack of a well-defined train of capillary waves and ripples might be due to strong viscous effects or even the numerical smoothing used to interpolate fluid properties from the front onto the fixed grid. The latter could also be responsible for the interface roughness shown in Fig. 7(f) in various places such as the tip of the jet and the crest of the parasitic wave where the interface is not a smooth curve.

Figures $8(a)-8(d)$ show the evolution in time of the spilling breaker corresponding to $\mathrm{Bo}=38$. The vorticity distribution as well as the velocity vectors are shown for each snapshot. In this case the role of surface tension is increased and induces the suppression of the jet formation. In comparison to the flow seen in Fig. 7, corresponding to a larger Bond number case, it can be observed that there is a relatively stronger shedding of vorticity in the water. In fact the curvature at the toe is very large which inhibits the flow from following the free surface shape unlike what was shown in Fig. 7. Figures $8(\mathrm{~b})-8(\mathrm{f})$ show flow separation and the development of a much stronger shear layer than was shown in Fig. 7. Consequently the amount of vorticity that is being produced and shed downstream is substantially larger in this case. The vorticity that is shed interacts with the free surface and in the process develops into independent well-defined vortical structures, which result into the ripples that are clearly visible at the free surface. These are eventually convected downstream. The maximum velocity is located at the bulge in the early stages of the wave evolution (at $\tau$ 


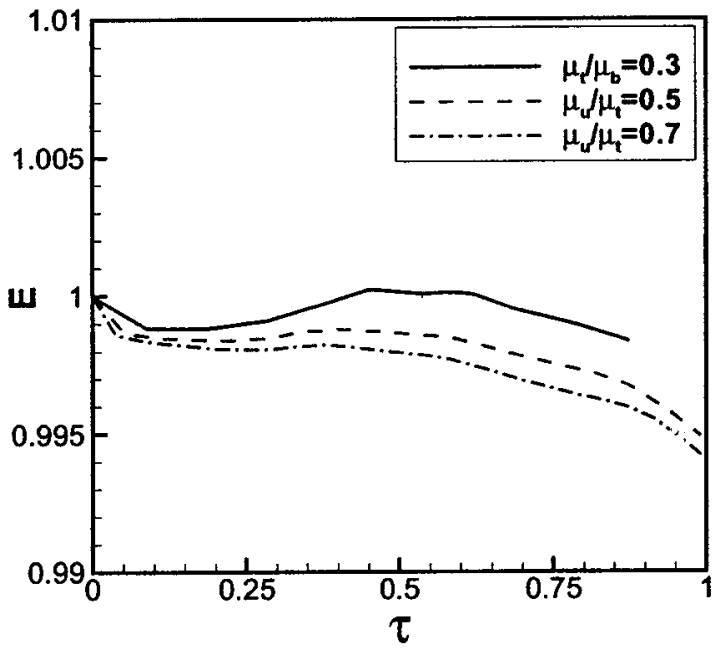

(a) Total Energy (E)

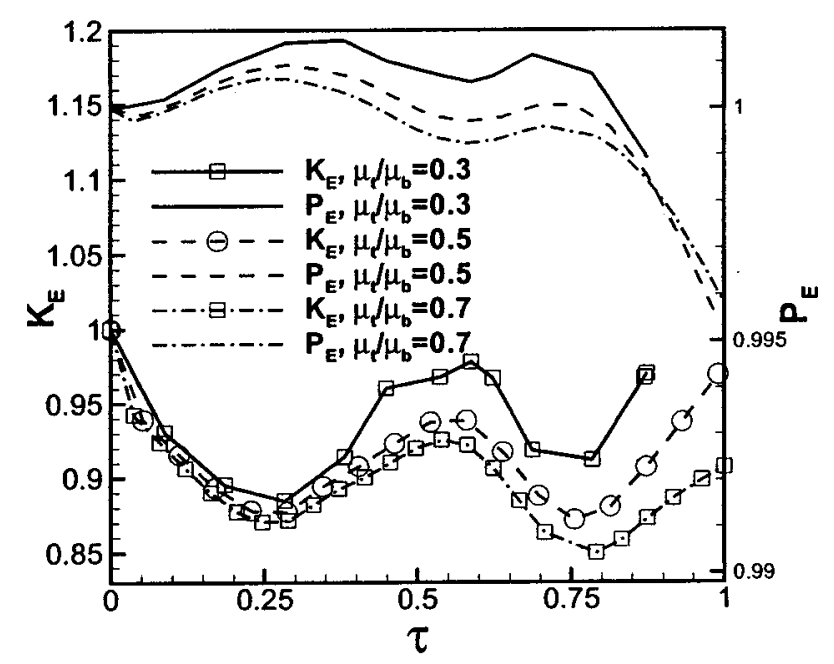

(b) Kinetic Energy $\left(K_{E}\right)$ and Potential Energy $\left(P_{E}\right)$

FIG. 19. Time evolution of total wave energy and wave potential and kinetic energies as a function of viscosity ratio $(\operatorname{Re}=350, \mathrm{Bo}=224$, density ratio $=0.028$, and $\mathrm{s}=0.5654$ ).

$=0.4202$ the maximum velocity is 2.3254$)$. At times corresponding to Figs. $8(\mathrm{~b})-8(\mathrm{~d})$ the maximum velocity takes place at the toe with corresponding maximum horizontal velocities components of $-1.7320,-1.7072$, and -1.534 . The maximum velocity takes place around $\tau=0.7577$ with a magnitude of 3.3249 corresponding roughly to the time when the first ripple appears. The second ripple is in its early stages in Fig. 8(f). After the appearance of the first ripple the points of maximum velocity take place at the trough of the first ripple. Such points correspond to locations $(0.3027,0.5273)$ and $(0.2539,0.5234)$ in Figs. $8(e)$ and $8(\mathrm{f})$, respectively, the corresponding velocity magnitudes increase from 2.0384 with a horizontal component of -1.9543 to 2.1725 and -2.1181 , respectively. In all Figs. 8(a)-8(f) the maximum vorticity is found at the toe also corresponding to the point of maximum curvature consistent with the observa- tions by Longuet-Higgins. ${ }^{30}$ Comparisons of Figs. 7 and 8 imply that surface tension effects can inhibit plunging. Also, the vorticity being shed into the water is exclusively due to the strong shear layer generated at the toe. While in the case of a plunging breaker the vorticity produced and shed into the water will be due to both the shear layer generated at the toe, even though less strong than in the latter case, and also air-entrapment once the jet impinges the interface.

Comparisons of the total energy corresponding to the cases presented in Figs. 4(a)-4(c) are shown in Fig. 9(a). The total wave energy is formed by the corresponding kinetic $\left(K_{E}\right)$, potential $\left(P_{E}\right)$ and surface tension energy $\left(S_{E}\right)$, defined as

$$
\begin{aligned}
& K_{E}=\frac{1}{2} \iint \rho \mathbf{u} \cdot \mathbf{u} d x d y, \\
& P_{E}=\iint \rho g y d x d y, \\
& S_{E}=\frac{\sigma}{\rho_{b} g \lambda^{2}}(\Gamma-1),
\end{aligned}
$$

where $\Gamma$ is the total arc length of the interface, and the integration is extended to the entire computational domain. The computations of the surface tension energy revealed a very small contribution to the total energy budget [of the order $\mathrm{O}\left(10^{-3}\right)$ ]. Figure 9 (b) shows the contributions of the kinetic and potential energy. The results presented in Fig. 9 are normalized by the corresponding values at $\tau=0$. The total energy initially decreases at about the same rate regardless of the Bond number up to approximately $\tau=0.05$. As expected the energy loss increases with Bond number afterwards, as the front becomes vertical. This takes place for all cases studied except that corresponding to $\mathrm{Bo}=597$ for which the total energy remains constant until the initial stages of jet overturning, around $\tau=0.5$, and decreases thereafter. In all cases the corresponding kinetic energy reaches a maximum around the time when the bulge is being formed, decreases quickly afterwards until the early stages of overturning when the kinetic energy reaches a minimum and the potential energy reaches a maximum. The effects of gravity decrease afterwards with a consequent drop in the total energy. The trends of the evolution of $K_{E}$ and $P_{E}$ for the higher Bond number are qualitatively and quantitatively very similar. For the low Bond number cases corresponding to 38 and 71 the trends of the evolution of $E, K_{E}$, and $P_{E}$ show some differences with respect to the higher Bond number cases. The total energy reaches levels higher than that corresponding to the initial condition. The level of $E$ remains then constant until the moment when the toe initiates its movement downstream, when $P_{E}$ starts to decrease at a faster rate. The appearance of ripples is associated with the final decrease of $K_{E}$ and $E$ in the later stages of the computation.

To further assess that the interface roughness, that was alluded to earlier and shown in Fig. 7(f), was not a consequence of the chosen initialization used in the computations, simulations were done using Fenton's ${ }^{61}$ third and fifth wave profiles. Comparisons were done with the results shown ear- 


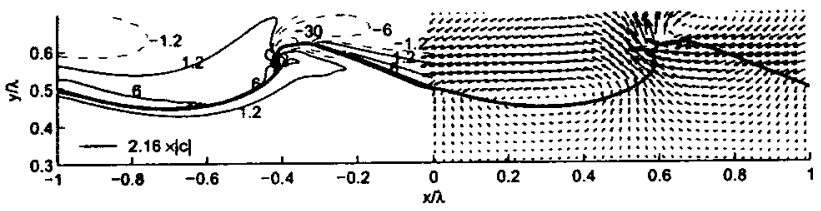

(a) $\tau=0.6611$

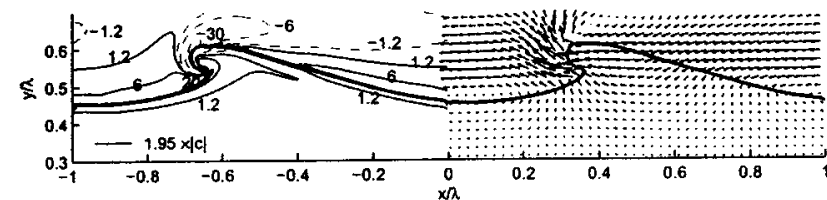

(b) $\tau=0.8568$

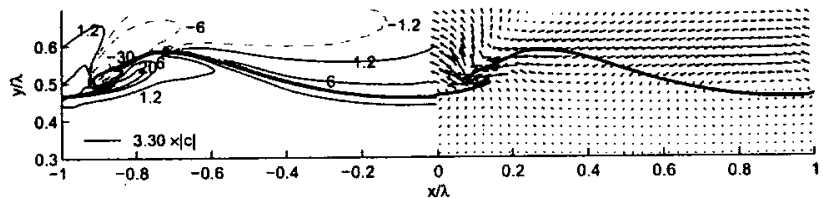

(c) $\tau=1.0230$

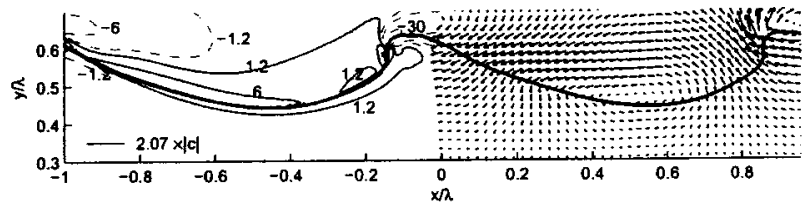

(d) $\tau=0.4446$

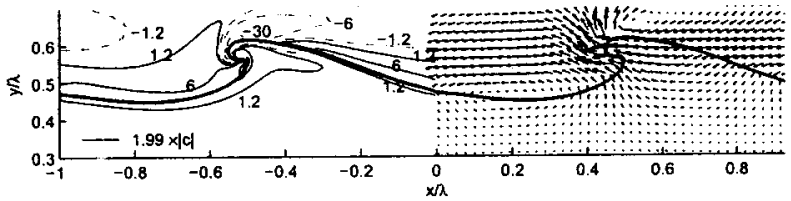

(e) $\tau=0.7372$

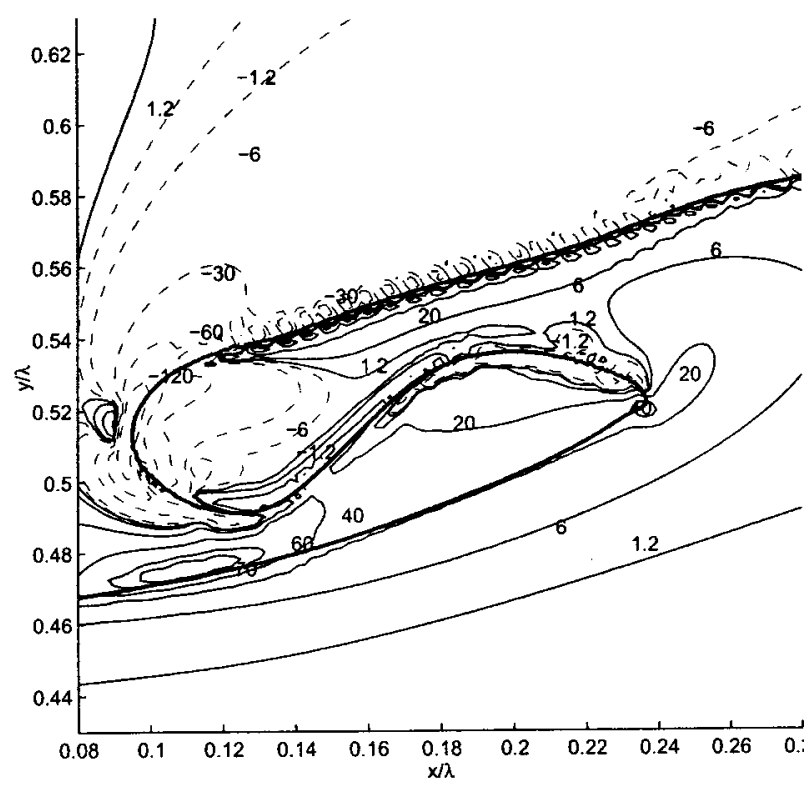

(f) $\tau=0.9713$

FIG. 20. Time sequence of free surface profiles showing the velocity field and the vorticity contours for initial wave slope $\varsigma=0.5507$ [left column, subplots (a) - (c)] and $\mathrm{s}=0.5654$ [right column, subplots $(\mathrm{d})-(\mathrm{f})$ ] $(\mathrm{Re}=350, \mathrm{Bo}=224$, density ratio $=0.028$, and viscosity ratio $=0.5)$. Positive vorticity contour $\left(\omega^{+}\right)---$, negative vorticity contour $\left(\omega^{-}\right) . \omega^{+}=20,6,1.2$ and $\omega^{-}=-30,-6,-1.2$.

lier in this section. Figures 10(a) and 10(b) shows a comparison of the overall wave evolution for the profiles corresponding to the second an fifth order waves (results corresponding to the third order wave are not shown due to their closer similarity to the second order wave results). As expected a change in the initial boundary condition substantially changes the evolution of the wave. Nonetheless, as Figs. 11(a) and 11(b) show the interface is not a smooth curve. These results lead the authors to conjecture that such interface irregularities are not tied with the choice of the initial profile but rather to the numerical methodology employed to smooth the physical properties from the interface onto the fixed grid, as mentioned earlier in this section.

\section{Reynolds number effects on wave breaking}

The effect of the Reynolds number was studied by conducting numerical experiments at $\mathrm{Re}=175$ and 500 in com- parison to the plunging breaker shown in Fig. 20, corresponding to $\mathrm{Bo}=224, \mathrm{Re}=350, \mathrm{~s}=0.5507$ and density and viscosity ratios 0.028 and 0.5 , respectively. The evolution of the free surface for both cases is shown in Fig. 12. As expected the higher the Reynolds number the less smooth the free surface becomes. No capillary waves, or ripples in the crest region are observed for Reynolds number 175 and 300. For the highest Reynolds number parasitic waves are observed in the neighborhood of the toe and crest. While the ripples seem to be short lived and their propagation downstream is fairly short, the roughness around the toe grows in amplitude very quickly and is damped as the overturning of the bulge takes place. The evolution of the toe shown in Fig. 12(a) indicates a progressive movement downstream with respect to the crest of the wave, up until overturning starts, when the toe movement stops. The behavior of the toe from Fig. 12(b) shows some resemblance to the main characteris- 


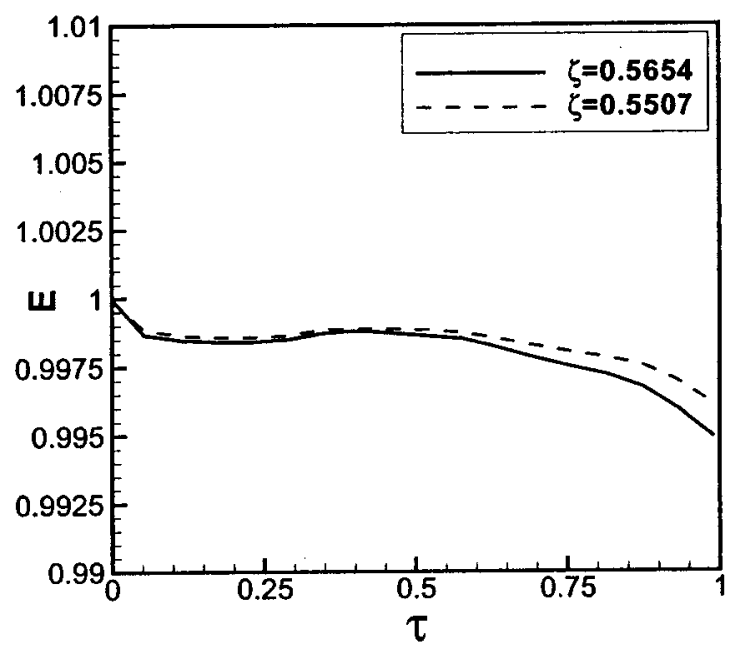

(a) Total Energy (E)

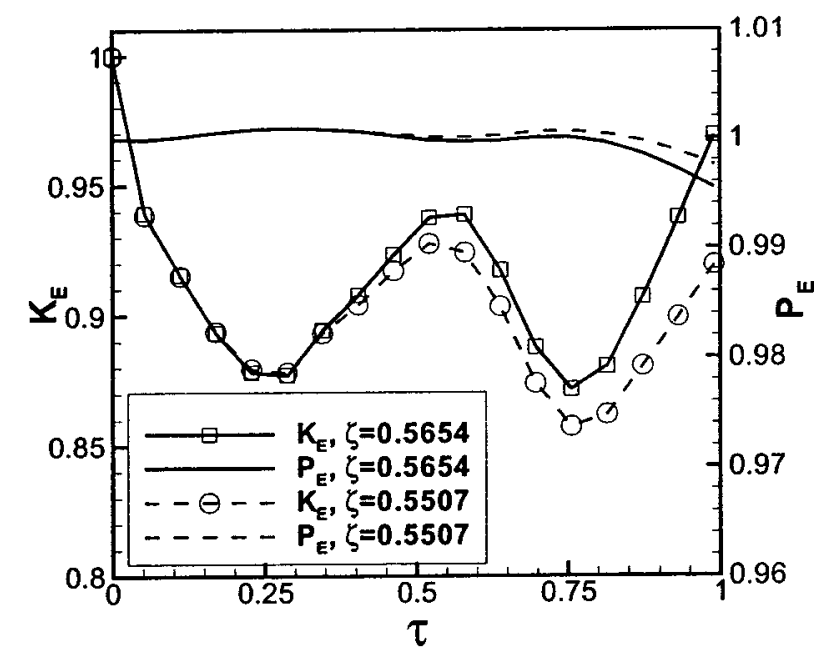

(b) Kinetic Energy $\left(K_{E}\right)$ and Potential Energy $\left(P_{E}\right)$

FIG. 21. Time evolutions of total wave energy and wave potential and kinetic energies as a function of initial wave slope $(\operatorname{Re}=350, \mathrm{Bo}=224$, density ratio $=0.028$, and viscosity ratio $=0.5$ ).

tics associated with surface tension effects. The toe moves downstream once the bulge is formed and seems to stop its motion during the initial stages of overturning. Figure 12(c) shows a snapshot corresponding to $\tau=0.9569$ for $\operatorname{Re}=500$, where the last traces of a single parasitic wave, with very small amplitude and large wavelength, is shown prior to jet impingement. Some interface roughness on the jet tip and a parasitic crest wave can also be seen in Fig. 12(c). The latter seems to be associated with the instability of the shear layer in the bulge as discussed for Fig. 7(f) in Sec. III C. Figure 13 shows the evolution of the main geometrical parameters representative of both waves, as defined in Fig. 5(a). As mentioned in Sec. III C the computations of the parameters $\left(y_{t}\right.$, $y_{c}$, and $y_{b}$ ) are done only at a few time steps. The results shown in Fig. 13(a) for $\mathrm{Re}=500$ are very similar to those corresponding to Fig. 5(c). The data shown for $\mathrm{Re}=175$ is very similar to that corresponding to the higher Reynolds number but, as expected, the evolution of the wave is slower in time. Figure 13(b) shows the evolution of the bulge thickness. The trends for both Reynolds numbers are very similar, even though the evolution corresponding to the flow with the lower Reynolds number is slower, also the thickness of the bulge is comparatively smaller to the higher Reynolds number flow. Qualitatively the data corresponding to $\mathrm{Re}=500$ is very similar to that presented in Fig. 5(d).

The time evolution of the total energy as well as that corresponding to the kinetic and potential energies is shown in Fig. 14. The lower the Reynolds number the larger the dissipation of energy. The evolution of energy in time corresponding to Reynolds numbers 500 and 350 is qualitatively very similar. On the other hand, in the case of $\mathrm{Re}=175$, the evolution of $E$ shows a progressive and quick decrease from $\tau=0$. The kinetic energy decreases up to the point when the front starts to become vertical, then increases up to $\tau=0.5$ when the toe begins to slide downstream and decreases thereafter.

\section{E. Density and dynamic viscosity ratios effects on wave breaking}

The impact of the density and viscosity ratios on wave breaking seems fairly intuitive. As the density of the top fluid increases, the ability of the wave to reach breaking will be progressively hampered. Such results can be seen from the free surface profiles shown in Fig. 15 for density ratios corresponding to 0.04 and 0.2 . These results can also be compared with the free surface evolution corresponding to a density ratio 0.02 shown in Fig. 3. The lower the density ratio the closer to a well-defined plunging breaker the wave evolution becomes. On the other hand, the results corresponding to the highest density ratio show that the breaker is completely suppressed. The evolution of each of the breakers shows that with less dense top fluids, the generation of the breaker becomes faster, and larger air entrapment takes place. The similarities in the quantitative and qualitative behavior of $E, P_{E}$, and $K_{E}$ are shown in Fig. 16.

The role of the dynamic viscosity ratio is assessed in Fig. 17 where the free surface evolution corresponding to viscosity ratios $0.3,0.5$, and 0.7 are shown. The simulations were done by systematically changing the dynamic viscosity of the top fluid while keeping all other parameters constant. The characteristics of the breakers are indeed influenced by this parameter. The evolution toward breaking becomes, as expected, slower and with less air entrapment with larger viscosity ratios. The differences in jet geometry as the flow gets closer to jet impingement are shown in Fig. 18, where a snapshot of the last time computed for each of the cases presented in Fig. 17 is shown along with the corresponding vorticity contours. The viscosity of the top fluid influences the speed of the breaker, as it can be inferred from Figs. 18(a)-18(c). The impingement of the jet takes place faster the less viscous the top fluid is. On the other hand, as the dynamic viscosity ratio increases for the same nondimensional parameters, the surface tension effects can eventually 
play an important role as seen in the pulling back of the interface shown in Fig. 18(c). The differences in entrainment are also clearly appreciable from Figs. 18(a)-18(c). The larger the dynamic viscosity ratio the more energy dissipation takes place as shown in Fig. 19.

\section{F. Initial wave slope effects on wave breaking}

The initial wave slope or steepness has an obvious impact on the wave evolution, presence of breaking, and consequent breaker type as it is well known. Quantitative and qualitative information is obtained for various breakers with different initial wave slopes. As the breaker becomes less powerful the wave evolves with a lower velocity and the angle of the plunging jet becomes less steep with respect to the forward face of the wave. In the limit the formation of the jet can be suppressed completely and replaced by a bulge at the crest of the wave, which then slides down the forward face of the wave.

Figure 20 shows snapshots of the time evolution of waves with the same nondimensional parameters other than wave slopes, which are $\varsigma=0.5507$ and 0.5654 , respectively. The wave evolution shown in Fig. 20 is very similar for both waves up to the time corresponding to that shown in Figs. 20(a) and 20(d), even though as expected the development of the less steep wave is slower. The maximum velocity corresponding to Fig. 20(a) is 2.1583 with a horizontal component of -1.1135 , while 2.0658 with -1.0849 corresponds to Fig. 20(d). At the last time steps shown in Figs. 20(c) and 20(f), the corresponding maximum velocities are 3.3096 and 2.6665 , respectively.

The lower fluid particle velocities corresponding to the breaker shown in Figs. 20(a)-20(c) are considerably reduced by comparison to the plunging jet shown in Figs. 20(d)20(f), as expected. Air entrapment is also practically suppressed for $\mathrm{s}=0.5507$ [Figs. 20(a) -20 (c)], and the large curvature at the jet root is responsible for the development of a strong shear layer by comparison to the results shown in Figs. 20(d)-20(f). The vorticity seems to extend over a larger and thicker region in the neighborhood of the interface by comparison to the results shown in Fig. 3 for the steeper wave. Vorticity is being shed in the water and ripples begin to appear in the free surface.

Comparisons of the total energy corresponding to the cases presented in Fig. 20 are shown in Fig. 21(a). Figure 21(b) shows the contributions of the kinetic and potential energies, respectively. The results presented in Fig. 21 are normalized by the initial values (at $\tau=0$ ) corresponding to the steeper wave. The total energy practically remains constant up to about $\tau=0.5$ when the energy starts to decrease much faster. This time corresponds roughly to the formation of the jet. Once the jet is formed there is a trade of energy between potential and kinetic energies, resulting into an increase of the latter to the expense of the former, as expected. Both cases for the two different initial wave slopes reflect the same trends, however for the steeper wave the energy dissipation associated with the generation and evolution of the plunger is larger as it is shown in Fig. 21.

\section{CONCLUSIONS}

In this paper a numerical study of wave breaking is conducted by solving the two-dimensional, incompressible, unsteady Navier-Stokes equations and using a hybrid front capturing/tracking technique for the numerical treatment of the immiscible interface. The interface is characterized by a jump in density and viscosity and is embedded in the computational domain. Numerical experiments are conducted to assess the role on wave breaking of each of the nondimensional parameters that govern the flow. This parametric study analyzes the influence of surface tension, Reynolds number, and density and viscosity ratios in the process of breaking and the type of breaker generated.

It is found that the increasing role of surface tension comes associated with a significant reduction in jet intensity and air entrapment. The lower the Bond number the more intense the shear layer generated at the toe of the bulge is. The vorticity generated by such shear layer is shed into the water and results into the generation of downstream propagating ripples. Their amplitude and wavelength increases in time except for the period coincident with the appearance of another ripple. The presence of such ripples is clearly found in this study for the lowest Bond number case studied. The first ripple appears at the time when the toe starts to slide down the front of the wave. No parasitic capillary waves are observed for that case. As the Bond number increases, though, traces of small amplitude short-lived parasitic capillary waves and ripples are found. They appear after overturning and the wave trains are formed by one or at most two of such waves.

As expected, the higher the Reynolds numbers the less smooth the free surface becomes. No capillary waves or ripples are observed for low Reynolds numbers, while parasitic waves in the neighborhood of the toe and the crest are observed for the largest Reynolds number studied. In this case the movement of the toe is found to be very similar to the case corresponding to the lowest Bond number, which as mentioned earlier showed the presence of downstream propagating ripples but no capillary waves.

The impact of density and viscosity ratios on wave breaking is more predictable. As the density of the top fluid increases, the ability of the wave to reach breaking is progressively hampered. The larger the dynamic viscosity ratio the more energy dissipation takes place, and the evolution toward breaking becomes slower and with less air entrainment.

${ }^{1}$ M. L. Banner and D. H. Peregrine, "Wave breaking in deep water," Annu. Rev. Fluid Mech. 25, 373 (1993).

${ }^{2}$ J. H. Duncan, "An experimental investigation of breaking waves produced by a towed hydrofoil," Proc. R. Soc. London, Ser. A 377, 331 (1981).

${ }^{3}$ W. K. Melville, "Wave modulation and breakdown," J. Fluid Mech. 128, 489 (1983).

${ }^{4}$ W. K. Melville, "The instability and breaking of deep-water waves," J. Fluid Mech. 115, 165 (1982).

${ }^{5}$ M. Y. Su, M. Bergin, P. Marler, and R. Myrick, "Experiments on nonlinear instabilities and evolution of steep gravity-wave trains," J. Fluid Mech. 124, 45 (1982).

${ }^{6}$ S. E. Ramberg, M. E. Barber, and O. M. Griffin, "Laboratory studies of steep and breaking deep water waves in a convergent channel," NRL Report No. 5610 (1985). 
${ }^{7}$ N. Ebuchi, H. Kawamura, and Y. Toba, "Fine structure of laboratory windwave surfaces studied using an optical method," Boundary-Layer Meteorol. 39, 133 (1987).

${ }^{8}$ W. K. Melville and R. J. Rapp, "The surface velocity field in steep breaking waves," J. Fluid Mech. 189, 1 (1988).

${ }^{9}$ S. P. Kjeldson and D. Myrhaug, "Kinematics and dynamics of breaking waves," Ships in Rough Seas, Part IV (Norwegian Hydrodynamic Laboratories, Trondheim, Norway, 1978).

${ }^{10} \mathrm{P}$. Bonmarin and Ramamonjiarsoa, "Deformation to breaking of deep water gravity waters," Exp. Fluids 3, 11 (1985).

${ }^{11} \mathrm{P}$. Bonmarin, "Geometric properties of deep-water breaking waves," J. Fluid Mech. 209, 405 (1989).

${ }^{12}$ R. J. Rapp and W. K. Melville, "Laboratory measurements of deep-water breaking waves," Philos. Trans. R. Soc. London, Ser. A 331, 735 (1990).

${ }^{13}$ D. J. Skyner, "A comparison of numerical predictions and experimental measurements of the internal kinematics of a deep-water plunging wave," in Water Wave Kinematics, edited by A. Torum and O. T. Gudmestad (Kluwer, Berlin, 1996), p. 491.

${ }^{14}$ M. Perlin, J.-H. He, and L. P. Bernal, "An experimental study of deep water plunging breakers," Phys. Fluids 8, 2365 (1996).

${ }^{15}$ A. H. Schooley, "Profiles of wind-created water waves in the capillarygravity transition region," J. Mar. Res. 16, 100 (1958).

${ }^{16} \mathrm{~K}$. Okuda, "Internal flow structure of short wind waves. Part I. On the internal vorticity structure," J. Oceanogr. Soc. Jpn. 38, 28 (1982).

${ }^{17}$ J. C. Lin and D. Rockwell, "Evolution of a quasi-steady breaking wave," J. Fluid Mech. 302, 29 (1978).

${ }^{18}$ J. H. Duncan, V. Philomin, M. Behers, and J. Kimmel, "The formation of spilling breaking," Phys. Fluids 6, S2 (1994).

${ }^{19}$ J. H. Duncan, V. Philomin, M. Behers, and J. Kimmel, "The formation of spilling breaking water wave," Phys. Fluids 6, 2558 (1994).

${ }^{20}$ J. H. Duncan, H. Qiao, V. Philomin, and A. Wenz, "Gentle spilling breakers: crest profile evolution," J. Fluid Mech. 379, 191 (1999).

${ }^{21}$ H. Qiao and J. H. Duncan, "Gentle spilling breakers: Crest flow-field evolution," J. Fluid Mech. 439, 57 (2001).

${ }^{22}$ M. S. Longuet-Higgins, "Progress toward understanding how waves break," 21st Symposium on Naval Hydrodynamics, Trondheim, Norway, 1996.

${ }^{23}$ M. S. Longuet-Higgins and E. D. Cocklet, "The deformation of steep surface waves on water. I: A numerical method for computation," Proc. R. Soc. London, Ser. A 350, 1 (1976).

${ }^{24}$ J. W. Dold and D. H. Peregrine, "An efficient boundary-integral method for steep unsteady water waves," Numerical Methods for Fluid Dynamics, edited by K. W. Morton and M. J. Baines (Oxford University, Oxford, 1986).

${ }^{25}$ D. G. Dommermuth, D. K. P. Yue, W. M. Lin, R. J. Rapp, E. S. Chan, and W. K. Melville, "Deep-water plunging breakers: A comparison between potential theory and experiments breaking waves," J. Fluid Mech. 189, 432 (1988)

${ }^{26}$ S. Grilli and I. A. Svendsen, "Computation of nonlinear wave kinematics during propagation and runup on a slope," Water Wave Kinematics, edited by A. Torum and O. T. Gudmestad (Kluwer, Berlin, 1990), p. 387.

${ }^{27}$ D. J. Skyner, C. Gray, and C. A. Greated, "A comparison of time-stepping numerical predictions with whole-field measurement in breaking waves," J. Fluid Mech. 315, 51 (1990).

${ }^{28} \mathrm{M}$. P. Tulin, "Breaking of ocean waves and downshifting," in Waves and Nonlinear Processes in Hydrodynamics, edited by J. Gure, B. Gjevik, and J. E. Weber (Dordrecht, Netherlands, 1996), p. 177.

${ }^{29}$ H. D. Ceniceros and T. Y. Hou, "Dynamic generation of capillary waves," Phys. Fluids 11, 1042 (1999).

${ }^{30}$ M. S. Longuet-Higgins, "Capillary rollers and bores," J. Fluid Mech. 240, 659 (1992)

${ }^{31}$ M. S. Longuet-Higgins, "Shear instability in spilling breakers," Proc. R. Soc. London, Ser. A 446, 309 (1994).

${ }^{32}$ R. C. Y. Mui and D. G. Dommermuth, "The vortical structure of parasitic capillary waves," J. Fluids Eng. 117, 355 (1995).

${ }^{33}$ H. D. Ceniceros, "The effects of surfactants on the formation and evaluation of capillary waves," Phys. Fluids 15, 245 (2003).

${ }^{34}$ J. J. Monagham, P. J. Bicknell, and R. J. Humble, "Volcanos, tsunamis and the demise of the minoans," Physica D 77, 217 (1994).

${ }^{35}$ G. Chen, C. Kharif, S. Zaleski, and J. Li, "Two-dimensional NavierStokes simulation of breaking waves," Phys. Fluids 11, 1 (1999).

${ }^{36}$ A. Iafrati and E. F. Campana, "Direct numerical simulation of surface tension dominated and non-dominated breaking waves," 24th Symposium on Naval Hydrodynamics, Fukuoka, Japan, 2002.

${ }^{37}$ F. H. Harlow and J. D. Shannon, "The splash of a liquid drop," J. Appl. Phys. 38, 10 (1967).

${ }^{38}$ F. H. Harlow and J. E. Welch, "Numerical calculation of time-dependent viscous incompressible flow of fluid with free surface," Phys. Fluids 8, $2182(1965)$

${ }^{39}$ A. J. Chorin, "Numerical solution of the Navier-Stokes equation," Math. Comput. 22, 745 (1968).

${ }^{40} \mathrm{R}$. Teman, "Approximate solution of Navier-Stokes equations by a fractionary step method 1," Arch. Ration. Mech. Anal. 32, 135 (1969).

${ }^{41}$ W. Tauber, S. O. Unverdi, and G. Tryggvason, "The nonlinear behavior a sheared immiscible fluid interface," Phys. Fluids 14, 2871 (2002).

${ }^{42}$ J. Adams, "MUDPACK: Multigrid Fortran software for the efficient solution of linear elliptic partial differential equations," Appl. Math. Comput. 34, 13 (1989).

${ }^{43}$ R. Scadovelli and S. Zaleski, "Direct numerical simulation of free-surface and interfacial flow," Annu. Rev. Fluid Mech. 31, 567 (1999).

${ }^{44}$ J. Glimm, "Nonlinear and stochastic phenomena-The grand challenge for partial differential equations," SIAM Rev. 33, 626 (1991).

${ }^{45}$ C. W. Hirt and B. D. Nichols, "Volume of fluid (VOF) method for the dynamics of free boundaries," J. Comput. Phys. 39, 201 (1981).

${ }^{46}$ M. Sussman, P. Smereka, and S. Osher, "A level set approach for computing solutions to incompressible two-phase flow," J. Comput. Phys. 114, 1469 (1994).

${ }^{47}$ M. Vogt and K.-J. Kang, "A level technique for solving 2-D free surface flow," 12th Workshop on Water Waves and Floating Bodies, Marseilles, 1997.

${ }^{48} \mathrm{M}$. Vogt, "A comparison between moving grid and a level set technique for solving 2-D free surface flows," ASME Fluids Engineering Summer Meeting, Vancouver, 1997

${ }^{49} \mathrm{~S}$. O. Unverdi and G. Tryggvason, "A front-tracking method for viscous, incompressible, multi-fluid flows," J. Comput. Phys. 100, 1 (1992).

${ }^{50}$ C. S. Peskin, "Numerical analysis of blood flow in heart," J. Comput. Phys. 25, 220 (1977).

${ }^{51}$ G. Tryggvason, B. Bunner, A. Esmaeeli, D. Juric, N. Al-Rawahi, W. Tauber, J. Han, S. Nas, and Y.-J. Jan, "Front-tracking method for the computations of multiphase flow," J. Comput. Phys. 169, 708 (2001).

${ }^{52}$ A. Esmaeeli and G. Tryggvason, "Direct numerical simulations of bubbly flows. Part 1. Low Reynolds number arrays," J. Fluid Mech. 377, 313 (1998); "Direct numerical simulations of bubbly flows. Part 2, Moderate Reynolds number arrays," ibid. 385, 325 (1999).

${ }^{53}$ J. Han and G. Tryggvason, "Secondary breakup of axisymmetric liquid drops. I. Acceleration by a constant body force," Phys. Fuids 11, 3650 (1999); "Secondary breakup of axisymmetric liquid drops. II. Impulsive acceleration," ibid. 13, 1554 (2001).

${ }^{54}$ M. S. Longuet-Higgins and E. D. Cocklet, "The deformation of steep surface waves on water. II: Growth of normal-mode instabilities," Proc. R. Soc. London, Ser. A 364, 1 (1978).

${ }^{55}$ M. Tanaka, "The stability of steep gravity-waves. 2," J. Fluid Mech. 156, 281 (1985).

${ }^{56}$ W. J. Jillians, "The superharmonic instability of Stokes waves in deep water," J. Fluid Mech. 204, 563 (1989).

${ }^{57}$ R. G. Dean and R. A. Darlymple, Water Wave Mechanics for Engineers and Scientists, 2nd ed. (World Scientific, Singapore, 1991).

${ }^{58}$ S. V. Patankar, C. H. Liu, and E. M. Sparrow, "Fully developed flow and heat transfer in ducts having streamwise-periodic variations of crosssectional area," Trans. ASME, Ser. K 99, 180 (1977).

${ }^{59}$ Y. Yang and G. Tryggvason, "Dissipation of energy by finite-amplitude surface waves," Comput. Fluids 27, 829 (1998).

${ }^{60}$ J. H. Duncan and A. A. Dimas, "Surface ripples due to steady breaking waves," J. Fluid Mech. 329, 309 (1996).

${ }^{61}$ J. D. Fenton, "A fifth-order Stokes theory for steady waves," J. Waterw., Port, Coastal, Ocean Eng. 111, 216 (1985). 